ARTICLE

Received 16 Apr 2014 | Accepted 30 Jun 2014 | Published 1 Aug 2014

DOI: $10.1038 /$ ncomms5563 OPEN

\title{
Selective suppression of excessive GluN2C expression rescues early epilepsy in a tuberous sclerosis murine model
}

N. Lozovaya $a^{1,2,3, \star}$, S. Gataullina ${ }^{1,2,3, \star}$, T. Tsintsadze ${ }^{1,2, \star}$, V. Tsintsadze ${ }^{1,2}$, E. Pallesi-Pocachard ${ }^{1,2}$, M. Minlebaev ${ }^{1,2,4}$, N.A. Goriounova ${ }^{1,2}$, E. Buhler ${ }^{1,2}$, F. Watrin ${ }^{1,2}$, S. Shityakov ${ }^{5}$, A.J. Becker ${ }^{6}$, A. Bordey ${ }^{7}$, M. Milh ${ }^{8}$, D. Scavarda ${ }^{8}$,

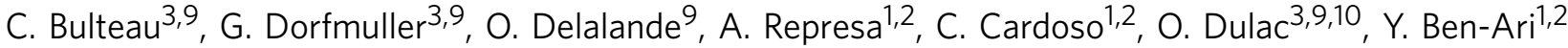
\& N. Burnashev ${ }^{1,2}$

Tuberous sclerosis complex (TSC), caused by dominant mutations in either TSC1 or TSC2 tumour suppressor genes is characterized by the presence of brain malformations, the cortical tubers that are thought to contribute to the generation of pharmacoresistant epilepsy. Here we report that tuberless heterozygote $\mathrm{Tsc}^{+/}-$mice show functional upregulation of cortical GluN2C-containing N-methyl-D-aspartate receptors (NMDARs) in an mTORdependent manner and exhibit recurrent, unprovoked seizures during early postnatal life $(<$ P19). Seizures are generated intracortically in the granular layer of the neocortex. Slow kinetics of aberrant GluN2C-mediated currents in spiny stellate cells promotes excessive temporal integration of persistent NMDAR-mediated recurrent excitation and seizure generation. Accordingly, specific GluN2C/D antagonists block seizures in $T s c 1^{+/-}$mice in vivo and in vitro. Likewise, GluN2C expression is upregulated in TSC human surgical resections, and a GluN2C/D antagonist reduces paroxysmal hyperexcitability. Thus, GluN2C receptor constitutes a promising molecular target to treat epilepsy in TSC patients.

\footnotetext{
${ }^{1}$ INSERM U901, INMED, Parc Scientifique et Technologique de Luminy 163, route de Luminy-BP 13, 13273 Marseille Cedex 09, France. ${ }^{2}$ UMR901, Aix-Marseille University, 58 Boulevard Charles Livon, 13284 Marseille, France. ${ }^{3}$ INSERM U1129; University Paris Descartes, CEA, Gif sur Yvette, 149 Rue de Sèvres, 75015 Paris, France. ${ }^{4}$ Laboratory of Neurobiology, Kazan Federal University, Kremlevskaya street 18, 420000 Kazan, Russia. ${ }^{5}$ Department of Anaesthesia and Critical Care, University of Würzburg, Josef-Schneider-Street 2, 97080 Würzburg, Germany. ${ }^{6}$ Department of Neuropathology, University of Bonn Medical Center, Sigmund Freud Street 25, D-53105 Bonn, Germany. ${ }^{7}$ Neurosurgery, and Cellular and Molecular Physiology Departments, Yale University School of Medicine, PO Box 208082, New Haven, Connecticut 06520-8082, USA. ${ }^{8}$ APHM, Department of Pediatric Neurosurgery and Neurology, CHU Timone, 264 Rue Saint-Pierre, 13385 Marseille Cedex 5, France. ${ }^{9}$ Department of Pediatric Neurosurgery, Foundation Rothschild, 29 Rue Manin, 75019 Paris, France. ${ }^{10}$ APHP, Necker Hospital, 149 Rue de Sèvres, 75015 Paris, France. * These authors contributed equally to this work. Correspondence and requests for materials should be addressed to N.L. (email: n_lozovaya@yahoo.com) or to N.B. (email: nail.burnashev@inserm.fr).
} 
T uberous sclerosis complex (TSC) is an autosomaldominant disease affecting multiple organ systems (brain, skin, kidney, heart and lung) and caused by germline mutations in one of the tumour suppressor genes, TSC1 or TSC2 (ref. 1). Inactivation of either of these genes leads to hyperactivation of the mammalian target of rapamycin (mTOR) pathway and promotes neuropathological abnormalities associated with $\mathrm{TSC}^{2-4}$. The most devastating clinical and pathological expressions of TSC involves the central nervous system, and includes malformative brain lesions, the cortical tubers, epilepsy, autism, cognitive impairment and glial tumours ${ }^{5}$. Epilepsy begins in infancy and is difficult to treat with $85-90 \%$ patients remaining with pharmacoresistant seizures ${ }^{5}$.

The molecular mechanisms governing epileptogenesis in TSC and the contribution of tuber formation have been intensively studied during the last decade and were subjects of intensive debates. Recent evidence indicates that gliomas are formed by biallelic TSC1 or TSC2 gene inactivation, reflecting a double-hit mechanism according to which a germline or a somatic mutation (likely occurring during development) affects the non-mutated allele, producing 'loss of heterozygosity'. However, loss of heterozygosity at either TSC gene is a rare event in tubers in human ${ }^{7}$, and although it may affect selected population of giant cells within cortical tubers, most of the cells in whole tuber sections have heterozygote mutations ${ }^{8}$.

Although correlations have been found between severity of the condition and the number of tubers ${ }^{9,10}$, and between topography of cortical tubers and type of epilepsy, it remains elusive whether tubers are intrinsically epileptogenic as some patients with numerous tubers have a benign condition, whereas some others, without tubers, have extremely severe epilepsy ${ }^{11-14}$. Furthermore, the epileptogenic zone may not be exactly superimposed to the lesion and includes some adjacent or, more rarely, remote areas ${ }^{12}$. This could explain why for approximately one-third of individuals who undergo epilepsy surgery, seizures persist after removal of the cortical tubers suspected to be epileptogenic ${ }^{9,10}$. Thus, it is not clear what mechanisms underlie epilepsy in perituberal region and in patients without evidence of cortical tubers or other dysgenetic features.

Numerous animal models of TSC have been generated in order to evaluate the mechanisms by which TSC genes loss results in the diverse pathological phenotypes. Mouse models generated using different brain-specific promoters and conditional alleles, in which both alleles of either $T s c 1$ or $T s c 2$ are lost in neurons or glia, display a severe neurological phenotype including morphological and clinical TSC features such as tuber-like structures, failure to thrive, frequent seizures and early mortality ${ }^{15-18}$. However, both heterozygous Tsc1 and Tsc2 knockout mice demonstrate behavioural and electrophysiological abnormalities and cognitive dysfunction in the absence of tubers or seizures ${ }^{4,19,20}$. This supports the concept that haploinsufficiency of $T s c 1$ or $T s c 2$ expression contributes significantly to the brain manifestations of TSC, although epileptic phenotype has not been reported so far for these mice.

Possible mechanisms of seizure generation in TSC could include changes in excitatory and inhibitory neurotransmitter function that may lead to abnormal neuronal synchronization and imbalance between excitation and inhibition ${ }^{21,22}$. Several reports have shown an increased expression of excitatory aminoacid binding sites in the epileptic cortex and altered ionotropic glutamate receptors expression patterns in human cortical tubers ${ }^{23-26}$. N-methyl-D-aspartate receptors (NMDARs) are putative candidates to explain the hyperexcitability of TSC neuronal networks. Slow decay kinetics of the currents mediated by NMDARs could facilitate synchronization in neuronal networks and thus exert proepileptic effects. However, the functional and proepileptic significance of NMDARs in epileptogenesis associated with TSC has not been explored.

In this study, we report that heterozygote $T s c 1^{+/-}$mice show functional upregulation of cortical GluN2C-containing NMDARs and exhibit spontaneous seizures associated with clinical manifestations during early postnatal life $(<\mathrm{P} 19)$ despite the absence of major morphological changes in the $\operatorname{brain}^{15,20}$. Selective inhibition of these receptors strongly disrupts spontaneous epileptiform activity in $T s c 1^{+}$- mice. Furthermore, electrophysiological and quantitative real-time reverse transcription polymerase chain reaction (RT-PCR) analysis of postsurgical tissue from TSC patients reveal upregulation of GluN2C subunits, indicating that these NMDAR subunits are instrumental in human TSC. Collectively, these observations suggest that an upregulation of GluN2C subunits is of paramount importance in the manifestation of epileptic phenotypes associated with TSC.

\section{Results}

Spontaneous seizures in $T s c 1^{+/-}$mice. To characterize functional abnormalities of haploinsufficient $T s c 1^{+/-}$mice, lacking major malformations ${ }^{15,20}$, in vivo intracortical electroencephalography (EEG) recordings in somatosensory S1 cortex of headrestrained non-anaesthetized $T s c 1^{+/}$mice at postnatal days P9-P33 were performed. Spontaneous recurrent seizures occurred in $77 \%$ of $\mathrm{Tscl}^{+}-$mice tested at P9-P18 (26 out of 34), but were not observed in $T s c 1^{w t}$ mice of the same age (Fig. 1 and Supplementary Figs 1 and 2). The seizures started 2-3 h after onset of EEG recordings and recurred as often as six per hour. Ictal discharges were often associated with screaming, oro-facial automatisms, head tremor, straub tail and tonic-clonic seizures (Supplementary Movie 1), followed by a quiet behaviour (Supplementary Movie 2).

Ictal EEG patterns started as high-frequency, low-amplitude activity that progressively evolved to high-amplitude regular polyspike trains involving all cortical layers. Subsequently, there were disruptions of the discharges with a reduction in EEG amplitude in cortex and appearance of high-amplitude rhythmic spike-wave trains in the hippocampus (Fig. 1a,b). Wavelet analysis showed an increase in high-frequency activity during ictal discharges (Fig. 1c). The contributions of $\delta, \gamma$ and fast ripple bands in power spectrum during discharges were significantly larger in neocortical layer 4 (L4) compared with layer 2/3 (L2/3; Fig. 1d, bottom panels). The amplitude and duration of discharges varied within litters with a mean amplitude of $615 \pm 18 \mu \mathrm{V}$ and a mean duration of $70.3 \pm 5 \mathrm{~s},(n=104$ seizures; $N=20$ mice; Fig. 1d, upper panels). Interestingly, epileptic phenotype was not observed in $T s c 1^{+/-}$mice at ages older than P19 (P19-P33, $N=10)$, indicating that this is a developmental insult.

The seizure onset as well as the peaks of averaged population spikes in L4 preceded those in L2/3 (Supplementary Fig. 3a, mean delay for the peaks was $8.0 \pm 1.3 \mathrm{~ms}, N=11$ mice). These observations and current source density (CSD) analysis suggest that epileptic activities in the cortex are initiated in granular cortical layer before spreading to supra- and infragranular layers (Supplementary Fig. 3b).

Qualitatively, similar results were obtained in coronal slices of $\mathrm{Tscl}^{+/}-$mouse brains, where intralaminar circuitry and horizontal recurrent connections were intact, but thalamocortical inputs were absent. In simultaneous whole-cell recordings from somatosensory cortical excitatory neurons in L4 and L2/3 spontaneous synchronized bursts were observed first in L4 and subsequently in L2/3 neurons with an average delay of 
a
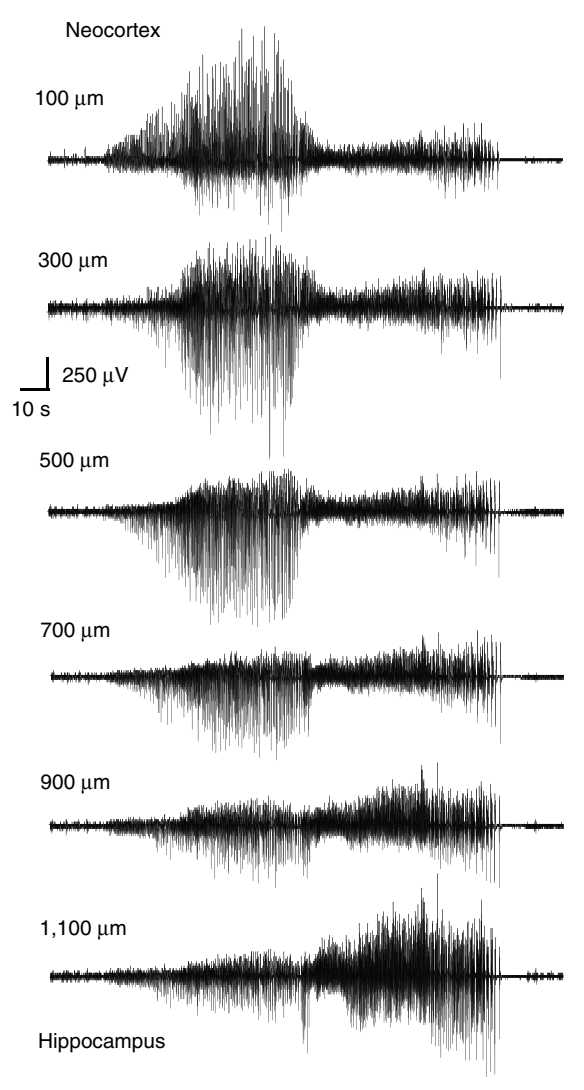

$1,200 \mu \mathrm{m}$
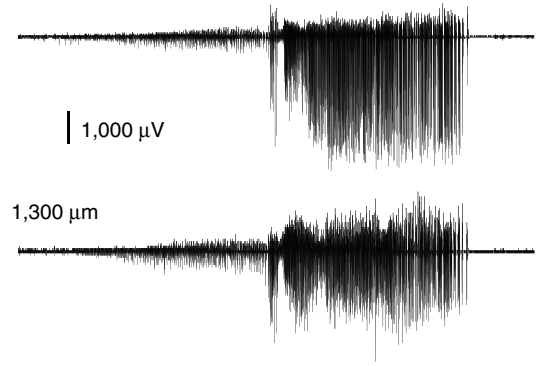

b
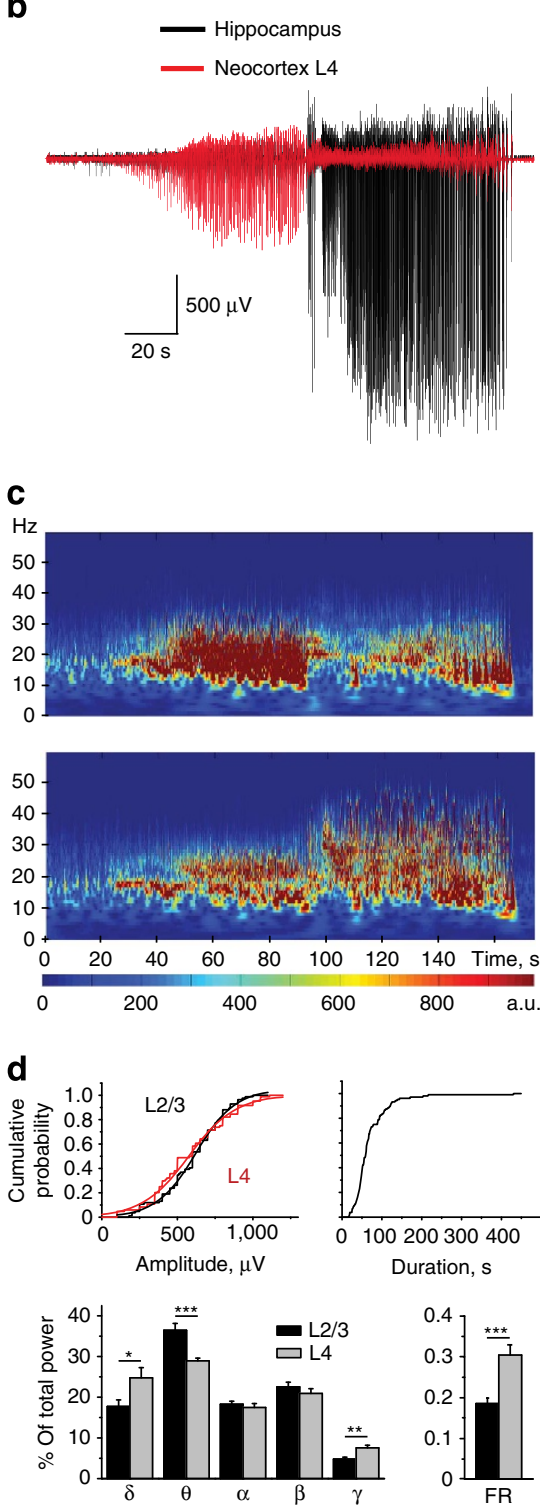

Figure 1 | Spontaneous seizures in Tsc1 ${ }^{+/-}$mice. (a) Intracortical EEG recorded using a 16-channel silicone probe in a head-restrained P16 Tsc1+/mouse. The upper channel corresponds to the superficial intracortical electrode placed at $100 \mu \mathrm{m}$ from the pia. Shown are epileptic discharges recorded at the depths indicated on the left of each trace. (b) Superimposed epileptic discharges in L4 of neocortex (red) and hippocampus (black). (c) Wavelet analysis during the ictal events for traces shown in $\mathbf{b}$. Upper panel: neocortex, lower panel: hippocampus. (d) Cumulative probabilities of seizures maximal amplitudes for L2/3 and L4 (upper left, $n=84$ seizures, $N=20$ mice) and durations (upper right, $n=104$ seizures, $N=20$ ). Seizure durations were the same for all layers, data for $\mathrm{L} 2 / 3$ are shown. Bottom: relative integral power of $\delta-(1-4 \mathrm{~Hz}), \theta-(4-8 \mathrm{~Hz}), \alpha-(8-12 \mathrm{~Hz}), \beta-(12-25 \mathrm{~Hz}), \gamma-(25-100 \mathrm{~Hz})$ and fast ripple (FR; $100-500 \mathrm{~Hz}$ ) band components of EEG in L2/3 ( $n=31$ seizures, $N=20$ ) and L4 ( $n=29$ seizures, $N=20$ ) revealed by Fourier transform analysis. All means \pm s.e.m.; ${ }^{\star} P<0.05,{ }^{\star \star} P<0.01,{ }^{\star \star \star} P<0.001$, two-sample two-tailed $t$-test.

$30.4 \pm 5.5 \mathrm{~ms}$ (61 synchronous bursts were analysed; Fig. 2a,b). Therefore, L4 neurons have a central role in neocortical epileptogenesis in $T s c 1^{+/-}$mice.

Upregulation of GluN2B and GluN2C/D NMDARs in Tsc1 ${ }^{+/-}$ mice. L4 is the main collector of sensory information and cortical 'hub' for intracolumnal information processing ${ }^{27}$. Recurrent activity triggered within the highly interconnected networks of L4 has been suggested to act to selectively amplify and redistribute transient high-frequency thalamo-cortical inputs ${ }^{28,29}$. What are the mechanisms underlying the increased integrative capacity of L4 neurons in $T s 1^{+/-}$mice? As NMDARs have an important role in L4 neuron integrative properties $^{30,31}$, we next examined whether slow NMDARmediated signalling was altered in $T s c 1^{+/-}$mice.

The L4-L4 connections are almost the only intracortical synaptic input that L4 spiny stellate cells (SSCs) receive ${ }^{32}$. This allows an estimation of the contribution of the slow NMDA component in isolated $\mathrm{L} 4-\mathrm{L} 4$ connections by measuring spontaneous activity from SSCs. To monitor the NMDARmediated current, we performed whole-cell recordings of spontaneous excitatory postsynaptic currents (sEPSCs) from L4 SSCs (Fig. 3a) and L2/3 pyramidal neurons (PNs) in coronal brain slices from $T s c 1^{+/}-$mice $(\mathrm{P} 14-\mathrm{P} 16)$ in voltage-clamp mode at $-50 \mathrm{mV}$. The decay kinetics of the composite sEPSCs was significantly slower in $T s c 1^{+/-}$than that in $T s c 1^{w t}$ mice both in L2/3 and L4 neurons (Fig. 3b,c), suggesting an increased 


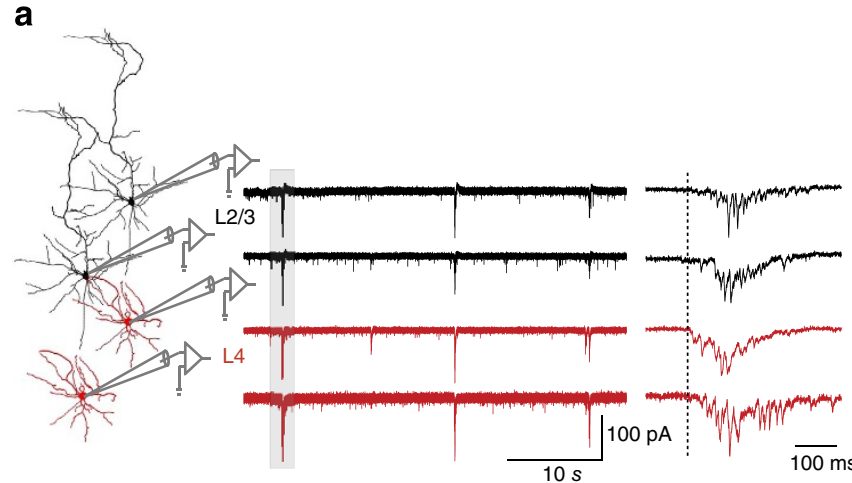

b
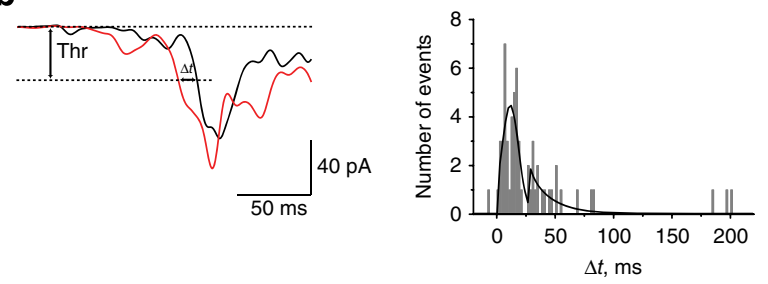

Figure 2 | Onset of spontaneous synchronous bursts in L4 preceded that in $\mathbf{L 2} \mathbf{3}$. (a) Middle: traces of whole-cell currents recorded at $V_{\mathrm{h}}=-70 \mathrm{mV}$ simultaneously from two PNs in L2/3 and two SSCs in L4 (shown schematically on the left) in neocortical slices from $\mathrm{Tsc}^{+/}{ }^{-}$mice. Right: expanded traces of the same bursts marked by grey box are shown. Vertical dotted line outlines bursts onset in L4. Note that onsets of the bursts in L4 precede those in L2/3. (b) Distribution of the onset delays $(\Delta t)$ between bursts recorded simultaneously from $L 2 / 3$ and $L 4$ neurons (61 synchronous bursts were analysed, $N=4$ mice). $\Delta t$ was measured from filtered $(0.5 \mathrm{kHz})$ events at the level of the burst threshold (Thr) set at $40 \mathrm{pA}$ as shown on the left. Events with amplitude $<40 \mathrm{pA}$ were not considered as the bursts. Fitting a Gaussian Function of $\Delta t$ distribution revealed at least two peaks with the most of the $\Delta t$ values peaking at $12 \mathrm{~ms}$.

contribution of NMDAR-mediated current and an upregulation of GluN2B, and/or GluN2C and GluN2D NMDAR subunits endowed with slow decay kinetics ${ }^{33}$. To quantify alterations in sEPSCs decay, we measured charge transfer normalized by the peak amplitude (see Methods section for details). In $T s c 1^{+/-}$ mice, normalized charges of sEPSC were $1.5 \pm 0.1$ in $\mathrm{L} 2 / 3$ and $2.55 \pm 0.16$ in L4 of those in $T s c 1^{w t}$ mice (L2/3: $n=27$ and 16 neurons; L4: $n=34$ and 29 neurons for $T s c 1^{+/-}$and $T s c 1^{w t}$ mice, respectively; two-sample, two-tailed $t$-test $T s c 1^{w t}$ versus $T_{s c 1} 1^{+/-}$in $\mathrm{L} 2 / 3 \quad P<5 \times 10^{-4}$, and in L4 $\left.P<1 \times 10^{-11}\right)$. Bi-exponential-weighted time constants of sEPSC decay $\left(\tau_{\mathrm{w}}\right)$ were in $T s c 1^{w t}$ mice $7.5 \pm 0.6 \mathrm{~ms}$ for $\mathrm{L} 2 / 3$ and $7.4 \pm 0.4 \mathrm{~ms}$ for $\mathrm{L} 4$, and in $T s c 1^{+/-}$mice $13.28 \pm 0.97 \mathrm{~ms}$ for $\mathrm{L} 2 / 3$ and $16.76 \pm 1.19 \mathrm{~ms}$ for L4 (two-sample, two-tailed $t$-test $T s c 1^{w t}$ versus $T s c 1^{+/-}$in L2/3 $P<2 \times 10^{-4}$, and in L4 $\left.P<8 \times 10^{-9}\right)$. Corresponding amplitudes of sEPSC in Tsc1 ${ }^{+/-}$mice were not significantly different from those in Tsc $1^{w t}$ mice in L4 (two-sample, two-tailed $t$-test, $P>0.7$ ), however, they were slightly increased in $\mathrm{L} 2 / 3$ (two-sample, two-tailed $t$-test, $P<0.07$; Supplementary Fig. 4).

To directly determine the involvement of NMDAR subtypes in prolongation of sEPSCs, we used specific GluN2B (Ro25-6981) and GluN2C/D (UBP141 (ref. 34), and DQP1105 (ref. 35)) antagonists (Supplementary Table 1). In L4 SSCs in Tsc1 ${ }^{+/}$ mice UBP141 and DQP1105, but not Ro25-6981, accelerated sEPSCs decay, restoring it to the values in $T s c 1^{w t}$ mice (Fig. 3d,f,i and Supplementary Table 2), suggesting an increased contribution of GluN2C/D but not GluN2B subunits. In $T s c 1^{+/-}$ mice, the normalized charge of sEPSC in L4 in the presence of
UBP141 $(10 \mu \mathrm{M})$ was $0.61 \pm 0.07\left(n=10, P<4 \times 10^{-4}\right.$, paired two-tailed $t$-test) and in the presence of DQP1105 $(10 \mu \mathrm{M})$ was $0.48 \pm 0.04\left(n=12, P<2 \times 10^{-7}\right.$, paired two-tailed $t$-test $)$ of that without drugs. Relative $\tau_{\mathrm{w}}$ values were $0.56 \pm 0.06$ for UBP141 $\left(P<5 \times 10^{-5}\right.$, paired two-tailed $t$-test $)$ and $0.41 \pm 0.04$ for DQP1105 $\left(P<3 \times 10^{-8}\right.$, paired two-tailed $t$-test $)$, respectively, of those without drugs. In $T s c 1^{w t}$ mice, sEPSCs decay kinetics was not affected by either drug (the normalized charge values were similar in the presence and absence of the drugs: $1.03 \pm 0.13$, $n=9, P>0.75$ for UBP141 and $1.06 \pm 0.07, n=12, P>0.4$ for DQP1105; paired two-tailed $t$-test). Correspondingly, the relative $\tau_{\mathrm{w}}$ values were $0.98 \pm 0.07, P>0.3$ for UBP141 and $1.07 \pm 0.07$, $P>0.7$ for DQP1105 (paired two-tailed $t$-test). Corresponding amplitudes of sEPSC for all sets were not significantly different (analysis of variance (ANOVA), $P>0.9$; Supplementary Fig. 4).

In addition, we performed recordings of miniature EPSCs (mEPSCs) in L4 cells in the presence of tetrodotoxin $(1 \mu \mathrm{M})$. Similarly to sEPSCs, the average decay kinetics of the late component in composite mEPSCs was significantly slower in $T s c 1^{+/-}$than that in $T s c 1^{w t}$ mice (Supplementary Fig. 5). Thus, in $T s c 1^{+/-}$mice, averaged normalized charge of mEPSC in L4 was $2.58 \pm 0.18$ of that in $T s c 1^{w t}$ mice $(n=10$ and 9 neurons, for $T s c 1^{+/-}$and $T s c 1^{w t}$ mice, respectively; two-sample, two-tailed t-test $T s c 1^{w t}$ versus $\left.T s c 1^{+/-} P<5 \times 10^{-7}\right)$. In $T s c 1^{+/-}$mice, the normalized charge of mEPSC and $\tau_{\mathrm{w}}$ in L4 in the presence of DQP1105 $(10 \mu \mathrm{M})$ were $0.45 \pm 0.07 \quad\left(n=8, \quad P<1.7 \times 10^{-4}\right.$, paired two-tailed $t$-test) and $0.55 \pm 0.03\left(n=9, P<3.2 \times 10^{-5}\right.$, paired two-tailed $t$-test), respectively of those without drugs. In $T s c 1^{w t}$ mice, mEPSCs decay kinetics were not affected by DQP1105: the normalized charge and $\tau_{\mathrm{w}}$ in the presence of DQP1105 were $0.96 \pm 0.14(n=7, P>0.75$, paired two-tailed $t$-test) and $1.13 \pm 0.13(n=8, P>0.48$, paired two-tailed $t$-test $)$, respectively, of those without drug. Corresponding amplitudes of mEPSC for all sets were not significantly different (ANOVA, $P>0.9$; Supplementary Fig. 5).

In contrast to the data in $\mathrm{L} 4$, the contribution of both GluN2B and GluN2C/D subunits was increased in L2/3 of Tsc1 ${ }^{+/-}$mice. Both Ro25-6981 and UBP141 accelerated sEPSC decay (Fig. 3e,h). In the presence of $1 \mu \mathrm{M}$ Ro25-6981, the normalized sEPSC charge in Tsc $1^{+/-}$mice was $0.79 \pm 0.05$ of control without Ro25-6981 ( $n=9, P<0.005$, paired two-tailed $t$-test). In $T s c 1^{w t}$ mice, this value was $1.03 \pm 0.09(n=6, P>0.75$, paired two-tailed $t$-test $)$. Similarly, corresponding numbers for UBP141 in L2/3 were $0.8 \pm 0.07$ of controls without the drug $(n=12, P<0.02$, paired two-tailed $t$-test) in $T s c 1^{+/-}$mice and $1.02 \pm 0.07(n=9, P>0.8$, paired two-tailed $t$-test) in $T s c 1^{w t}$ mice. However, the interpretation of UBP141 effects in L2/3 is complicated by the fact that at a concentration of $10 \mu \mathrm{M}$ it partially affects also GluN2B receptors (Supplementary Table 1). The amplitudes of sEPSCs in the presence of antagonists were not significantly different from control values for all experimental sets (ANOVA, $P>0.15$; Supplementary Fig. 4).

Therefore, there is an increased contribution of GluN2C/D subunits in L4, but of both GluN2B and GluN2C/D subunits in $\mathrm{L} 2 / 3$ in $T s c 1^{+/-}$mice when compared with naive $T s c 1^{\mathrm{wt}}$ mice.

To determine whether upregulation of the GluN2C/DNMDAR component in L4 is a direct consequence of enhanced mTOR signalling caused by Tsc1 inactivation, we performed experiments in $T s c 1^{+/-}$mice chronically treated with the mTOR inhibitor rapamycin (see Methods section). Recordings of sEPSC from SSCs in L4 in rapamycin-treated $T s c 1^{+/-}$mice (P14-P16) showed the absence of the UBP141-sensitive component (Fig. 3d,g,j). In rapamycin-treated $T s c 1^{+/-}$mice, the normalized charge of sEPSCs in SSCs in the presence of UBP141 $(10 \mu \mathrm{M})$ was $1.05 \pm 0.11(n=12, P>0,6$, paired two-tailed $t$-test $)$ of that without the drug. Furthermore, normalized charge of 
a

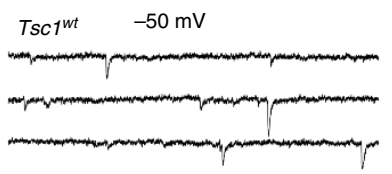

Tsc1 ${ }^{+/-}$
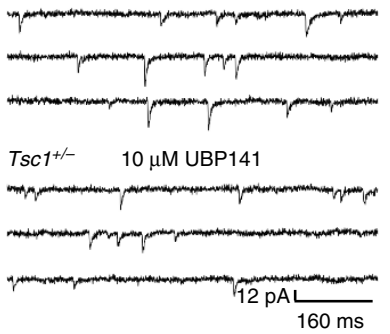

e

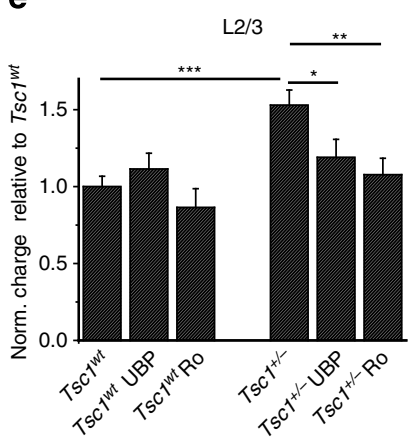

h

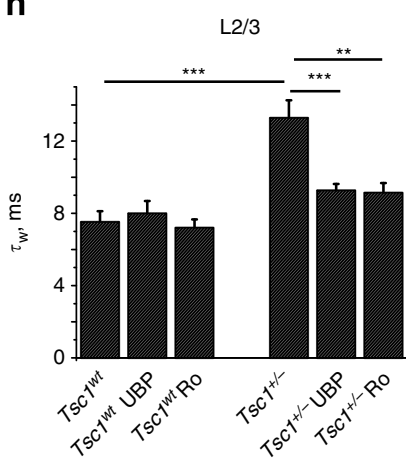

b

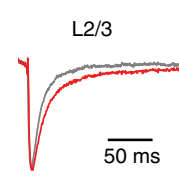

d

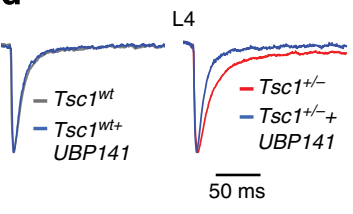

f

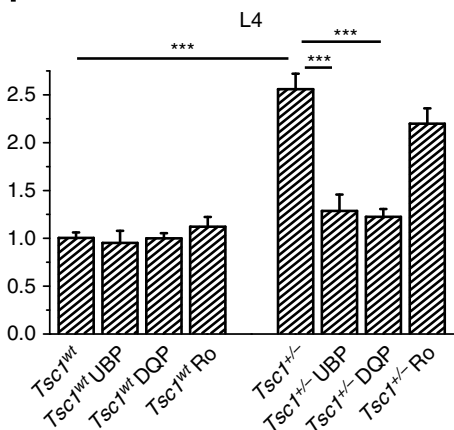

i

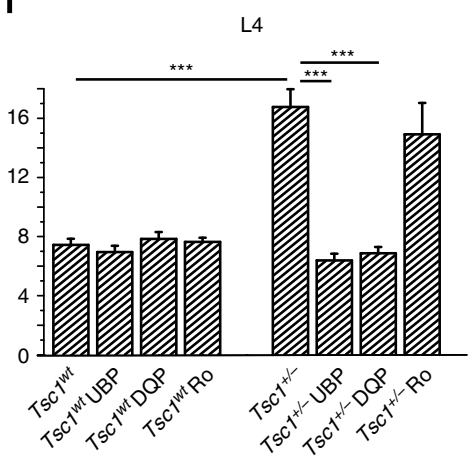

C
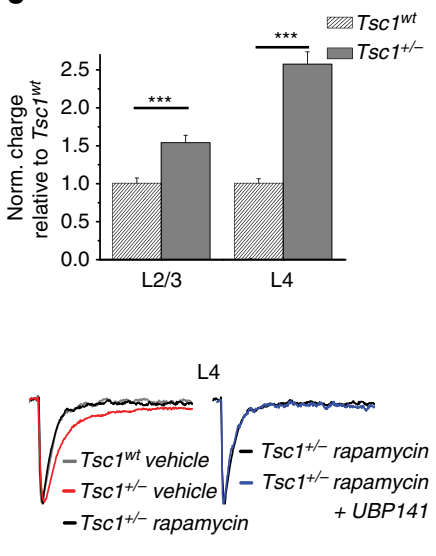

g

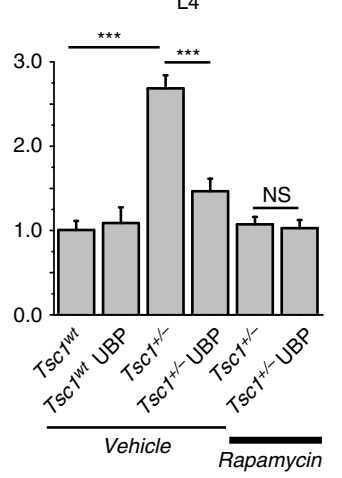

j

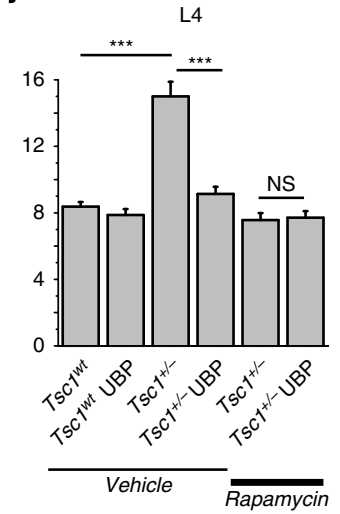

Figure 3 | Layer-specific functional upregulation of GluN2B and GluN2C/D subunits containing NMDA receptors in Tsc1 ${ }^{+/}$- mice. (a) Representative traces of spontaneous activity recorded in whole-cell mode at holding potential $-50 \mathrm{mV}$ from SSCs in L4 in neocortical coronal slices from Tscitt, $\mathrm{Tsc1}^{+/-}$mice and in $\mathrm{Tsc}^{+} /{ }^{-}$mice in the presence of UBP141 $(10 \mu \mathrm{M})$. (b) Grand averages of normalized (Norm.) and superimposed traces of sEPSC recorded from PNs in L2/3 (left) or SSCs in L4 (right) in $T s c 7^{w t}$ and $T s c 1^{+/}-$mice at $-50 \mathrm{mV}$. For each neuron, original traces from individual experiments were aligned based on the starts of their rising phases and averaged. These averaged traces from individual experiments were normalized and averaged to form grand average traces shown. Pooled data from 16 neurons for Tsc $7^{w t}(N=5$ mice $)$ and 27 neurons for $T s c 7^{+/-}$in $L 2 / 3(N=8)$ and from 29 neurons for $T s c 7^{w t}(N=6)$ and 34 neurons for $T s c 1^{+/-}$in $L 4(N=10)$. (c) Summary data for normalized charges of sEPSC in $L 2 / 3$ and $\mathrm{L} 4$ in $T s c T^{w t}$ and in $T s c 1^{+/-}$mice (relative to $T s c 7^{w t}$ ). (d) Left two panels: superimposed grand average of normalized traces of $s E P S C$ recorded in $\mathrm{L} 4$ in $\mathrm{Tsc}^{\mathrm{wt}}$ and $\mathrm{Tsc}^{+/} /-$mice in control and in the presence of UBP141. Right two panels: superimposed grand average of normalized traces of sEPSC recorded in L4 in Tsc1 ${ }^{+/-}$and Tsc $7^{w t}$ mice pretreated with either vehicle or rapamycin. (e,h) Summary data for the effects of UBP141 and Ro25-6981 ( $1 \mu \mathrm{M})$ on normalized charges of sEPSC (e) and weighted time constant, $\tau_{\mathrm{w}}$ (h) of sEPSC decay in L2/3 in TsC $7^{w t}$ and in Tsc ${ }^{+} /-$mice. (f,i) Summary data for the effects of UBP141, DQP1105 $(10 \mu \mathrm{M})$ and Ro25-6981 on normalized charges of sEPSC (f) and $\tau_{\mathrm{w}}(\mathbf{i})$ in L4 in Tsc ${ }^{w t}$ and in $\mathrm{Tsc1}^{+/-}$mice. (g,j) Summary data for the effects of UBP141 on normalized charges of SEPSC $(\mathbf{g})$ and $\tau_{\mathrm{W}}(\mathbf{j})$ in $L 4$ in $T s c 7^{W t}$ and in Tsc1 ${ }^{+/}-$mice pretreated with either vehicle or rapamycin. All means \pm s.e.m. All data sets were analysed using one-way ANOVA followed by Fisher's Least Significant Difference (LSD) post hoc test (see Supplementary Table 2 for statistics); ${ }^{\star} P<0.05,{ }^{\star \star} P<0.01$, ${ }^{\star \star \star} P<0.001$. NS, not significant, 
a

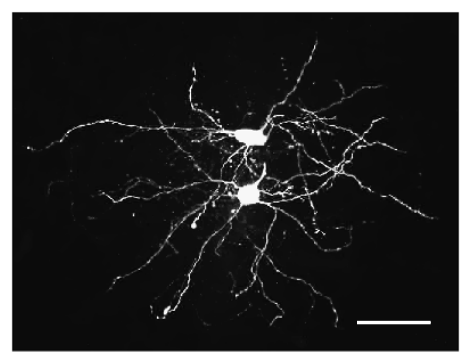

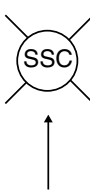<smiles>CC1CCC2CCC3CCC(C1)C23</smiles>

b
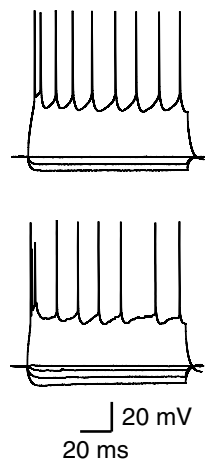

C
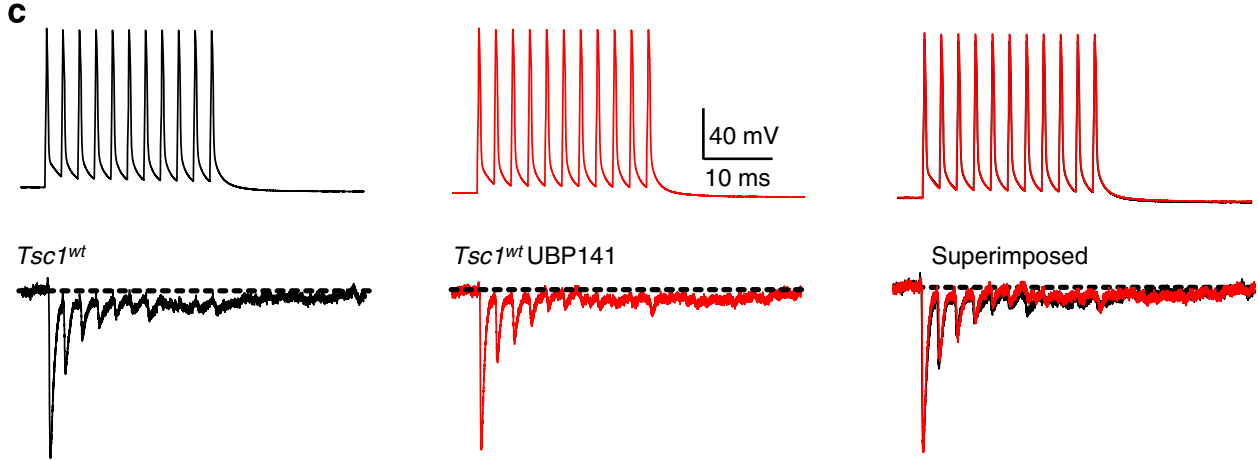

d
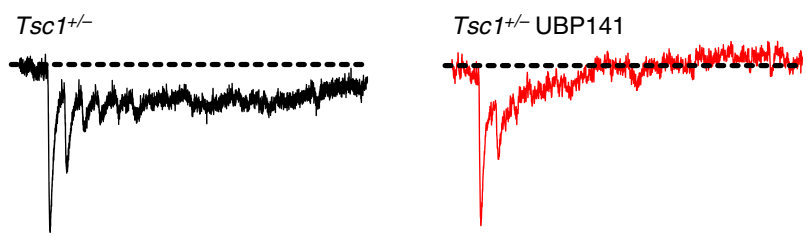

e

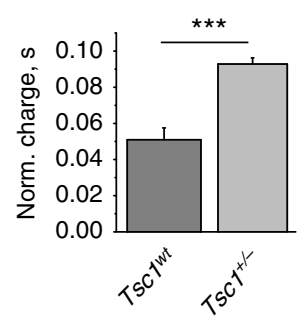

f

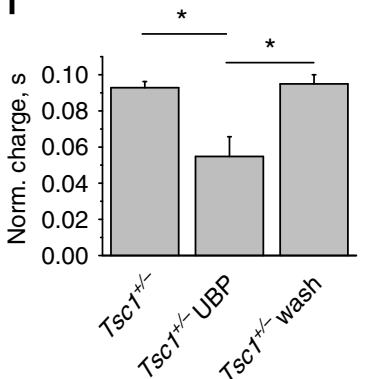

Figure 4 | Enhanced temporal integration of synaptic responses in synaptically coupled SSCs in L4 in Tsc1 ${ }^{+}$- mice. (a) Fluorescence images from two SSCs (left) and the configuration of a paired recordings (right). Scale bar, $50 \mu \mathrm{m}$. (b) Representative action potential firing patterns of both, postsynaptic (top) and presynaptic (bottom) SSCs recorded in current clamp mode. (c,d) Representative averaged normalized by the first peaks in the trains traces of EPSCs recorded in voltage-clamp mode in pairs of SSCs in L4 (averaged 25 sweeps) from Tsc1 ${ }^{\text {wt }}$ (c, bottom panels) and Tsc1 ${ }^{+} /-$ (d) mice induced by train stimulation ( 11 stimuli at $40 \mathrm{~Hz}$ ) in presynaptic cell in the absence (left panels, black traces) or presence (middle panels, red traces) of UBP141 $(10 \mu \mathrm{M})$. Top traces in c represent averaged superimposed presynaptic SSC action potentials in control and in the presence of UBP141. Right panels: the traces in the absence and in the presence of UBP141 are superimposed. (e) Normalized charge transfer of the train-EPSCs estimated from normalized by amplitude of the first peak currents is increased in $T s c T^{+/-}\left(n=5\right.$ cell pairs, $N=4$ mice) compared with $T s c T^{W t}$ mice $(n=7$ cell pairs, $N=4), P<0.0005$, two-tailed $t$-test. (f) UBP141 decreases the contribution of NMDAR-mediated EPSCs to the postsynaptic summation of EPSCs in synaptically coupled SSCs in Tsc1 ${ }^{+/-}$mice. Normalized charge transfer of the train-EPSCs from Tsc ${ }^{+/-}$mice in control $(n=5 \mathrm{cell}$ pairs, $N=4)$, in the presence $(n=4$ cell pairs, $N=4)$ and after washout of UBP141 $(n=3$ cell pairs, $N=3)$. Tsc $1^{+/}-$versus Tsc $1^{+/-}$in the presence of UBP141, $P<0.03$, two-tailed $t$-test). ( $(\mathbf{g})$ Normalized charge transfer of the train-EPSCs is unaltered in the presence of UBP141 in Tsc $7^{w t}$ mice $(n=6$ cell pairs, $P>0.44$, two-tailed $t$-test). All means \pm s.e.m., ${ }^{\star} P<0.05$, ${ }^{\star \star \star} P<0.001$. NS, not significant. 
sEPSCs in rapamycin-treated $T s c 1^{+/-}$mice was not significantly different from that of vehicle-treated $T s c{ }^{\mathrm{Wt}}$ mice $(1.05 \pm 0.08$ of that in vehicle-treated $T s c 1^{w t}$ mice, $n=14$ for $T s c 1^{+/-}, n=10$ for $T s c 1^{w t}$, two-sample two-tailed $t$-test $P>0.75$; Fig. $3 \mathrm{~g}$ ). This finding indicates a crucial role of mTOR signalling in functional upregulation of GluN2C/D-containing NMDARs in $\mathrm{Tscl}^{+/-}$mice.

Interestingly, in L4 fast-spiking (FS) interneurons neither UBP141 nor Ro25-6981 altered sEPSCs decay in both $T s c 1^{\text {wt }}$ and $T s c 1^{+/-}$mice (Supplementary Fig. 6), suggesting that NMDARmediated currents in inhibitory neurons remain intact.

To further determine the functional effects of UBP141 in $T s c 1^{+/-}$mice, we tested its actions on the amplitude and frequency of $\alpha$-amino-3-hydroxy-5-methyl-4-isoxazolepropionic acid receptor (AMPAR)-mediated sEPSCs. UBP141 altered neither the amplitude nor the kinetics of AMPAR-mediated sEPCS recorded at $-80 \mathrm{mV}$ in L4 SSCs (Supplementary Fig. 7a,b). The frequency of sEPSCs recorded at $-80 \mathrm{mV}$ in L4 SSCs was not different from that in $T s c{ }^{w t}$ mice. However, in L2/3 PNs it was significantly higher than that of $T s c 1^{w t}$ mice (Supplementary Fig. 7c), and was reduced by bath application of UBP141 in $T s c 1^{+/-}$(Supplementary Fig. 7e), but not in $T s c 1^{w t}$ (Supplementary Fig. 7d) mice. Therefore, in Tsc1 $1^{+/-}$ mice L4 GluN2B/C/D channels presynaptic to L2/3 PN could contribute to increase the activity of the latter.

mTOR-dependent upregulation of the slow NMDAR subunits was confirmed by quantitative RT-PCR revealing 2.4-fold elevation of GluN2C mRNAs $(P<0.005$, two-tailed $t$-test $)$ in the neocortex of $T s c 1^{+/-}$mice $(N=5)$ compared with agematched $T s c 1^{w t}(N=5)$ mice (Supplementary Fig. 8).

Thus, the contribution of GluN2C-containing NMDARs with slow kinetics is increased in neocortical L4 and L2/3 excitatory neurons in mice with haploinsufficient Tscl mutation. Similar alterations in SEPSC kinetics were observed in 'double-hit' Tsc1 mutant mice. In a recent study, using in utero electroporation and relying on the «second-hit» mutation concept ${ }^{36}$, Bordey and coworkers ${ }^{15}$ generated a $T s c 1^{-1-}$ animal model with the hallmark of human TSC, namely the tubers. We used this model to test the impact of a «second-hit» mutation on functional upregulation of slow NMDARs. To do that, $T s c 1^{f l x / m u t}$ mice (as well as $T s c 1^{f l x / w t}$ ) were injected in utero at embryonic day 16 (E16) with pCAGmRFP alone or combined with pCAG-Cre:GFP to induce, after electroporation, deletion of the floxed Tsc1 gene in a subset of neurons. The fluorescence of the monomeric red fluorescent protein (mRFP) plasmid allows identification of electroporated neurons and green fluorescent protein (GFP) for detection of neurons containing pCAG-Cre. Whole-cell recordings in neocortical slices from $\mathrm{mRFP}^{+} / \mathrm{GFP}^{+}$neurons of $T s c 1^{f x / m u t}$; pCAG-Cre and Tsc $f^{f l x / w t}$; pCAG-Cre conditional knockout mice (hereafter referred to as Tsc1 ${ }^{\text {null }}$ and Tscl haplo neurons, respectively) at $-50 \mathrm{mV}$ revealed enhanced contribution of slow UBP141-sensitive NMDAR-mediated components in sEPSC compared with those from control mRFP ${ }^{+}$neurons of $T s c f^{f l x / w t}$ mice, in the same manner as in non-electroporated heterozygote $T s c 1^{+/-}$mice. Therefore, GluN2C/D-mediated currents are present in both Tsc1 haplo and «double-hit» Tsc1 $1^{\text {null }}$ neurons. Importantly, the extent of slow NMDARs contribution was the same for the electroporated neurons with heterozygote and homozygote Tsc1 mutations (Supplementary Fig. 9).

Slow NMDAR-mediated signal determines temporal integration. To test temporal summation at $\gamma$-band frequency, we performed simultaneous whole-cell recordings from pairs of synaptically coupled SSCs in L4 of somatosensory cortex in slices from $T s c 1^{+/-}$and $T s c 1^{w t}$ P14-P16 mice. The majority of interconnected excitatory neurons were SSCs with an asymmetrical dendritic arborization largely confined to L4 and characteristics action potentials firing pattern (Fig. 4a,b). In concordance with the slower NMDAR-mediated component of synaptic transmission in $T s c 1^{+/-}$mice, recordings revealed a significantly higher extent of temporal integration compared with $T s c{ }^{w t}$ mice when measuring EPSCs evoked by stimulation of presynaptic cells with a train of action potentials. Normalized charge transfer of the train-EPSCs estimated from normalized by the amplitude of the first peak currents was $0.093 \pm 0.004 \mathrm{~s}$ in $\mathrm{Tsc}^{+/}-(n=5)$ and $0.050 \pm 0.006 \mathrm{~s}(n=7)$ in $T s c 1^{w t}$ mice, respectively, $(P<0.0005$, two-tailed $t$-test; Fig. $4 \mathrm{c}-\mathrm{e})$. Furthermore, recordings in $\mathrm{Tscl}^{+/-}$mice demonstrated significantly increased contribution of UBP141-sensitive NMDAR-mediated train-EPSCs to the postsynaptic summation of EPSCs but not in $T s c 1^{w t}$ mice (Fig. 4d,f,g). In the presence of UBP141, the normalized sEPSC charge in $T s c 1^{+}{ }^{-}$mice was $0.57 \pm 0.11$ of that without UBP141 ( $n=4, P<0.03$, paired two-tailed $t$-test). In $T s c l^{w t}$ mice, this value was $1.06 \pm 0.07(n=6, P>0.44$, paired two-tailed $t$-test). Thus, abnormal slowing of the NMDARmediated current kinetics increases temporal integration within recurrent network in $\mathrm{L} 4$ of $T s c 1^{+/-}$mice.

GluN2C/D antagonists reduce epileptogenecity in $T s c 1^{+/-}$ mice. The critical role of L4 neurons in increased temporal integration and seizure generation suggests that the selective blockade of long-lasting GluN2C/D subunits containing NMDAR-mediated currents may have antiepileptic effects. To test this possibility, we first used microelectrode array extracellular recordings in acute coronal neocortical slices taken from P15 Tsc1 ${ }^{+/-}$mice. Spontaneous discharges lasting for up to $10 \mathrm{~s}$ were recorded in L2/3 and L4. UBP141 $(10 \mu \mathrm{M})$ selectively reduced the amount of long-lasting epileptiform episodes, without altering the number of interictal bursts (duration $\leq 500 \mathrm{~ms}$; Supplementary Fig. 10).

We next tested the antiepileptic actions of UBP141 and DQP1105 in vivo by intraperitoneal (i.p.) injections of the drugs (75 and $28 \mathrm{mg} \mathrm{kg}^{-1}$, respectively) to $T s c 1^{+/-}$mice. These values were the lowest effective doses identified by testing increasing doses of these compounds (see Methods section for details). In three of six mice tested, the seizures were completely stopped $\sim 40$ min after i.p. injection of UBP141, and in the remaining mice $(n=3)$ there was a seizure-free period lasting $109 \pm 39 \mathrm{~min}$ (Fig. 5a,b). I.p. injection of DQP1105 stopped seizures on average for $72.6 \pm 5.5 \mathrm{~min}$ in four out of six mice, and seizures were completely stopped in two mice (Fig. 5c). In contrast, recurrent epileptic discharges persisted up to $7 \mathrm{~h} \mathrm{in} T s \mathrm{Tl}^{+/-}$mice that did not receive the antagonists or were i.p. injected with vehicle (Fig. 5d,e). Therefore, selective antagonists of GluN2C/Dcontaining receptors have in vivo and in vitro antiepileptic actions in $T s c 1^{+/-}$mice.

Importantly, after UBP141 injection a basal activity remained unaltered, in particular, at $\gamma$-frequency band (Supplementary Fig. 11), known to be enhanced by common NMDAR antagonists ${ }^{37}$. This might indicate that used dosage of UBP141 corresponds to the concentration in the brain to be within the selectivity range for GluN2C/D. The used dosage of DQP1105 $\left(28 \mathrm{~m} \mathrm{~kg}^{-1}\right)$ according to in silico prediction tools appears to be also within the selectivity range for GluN2C/D receptors (see Methods section for details and Supplementary Table 1). The direct assessment of the distribution of both drugs in the brain requires further studies.

Collectively, our observations on the $T s c 1^{+/-}$animal model suggest that seizures are generated intracortically owing to an upregulation of GluN2C receptors in recurrent connections 


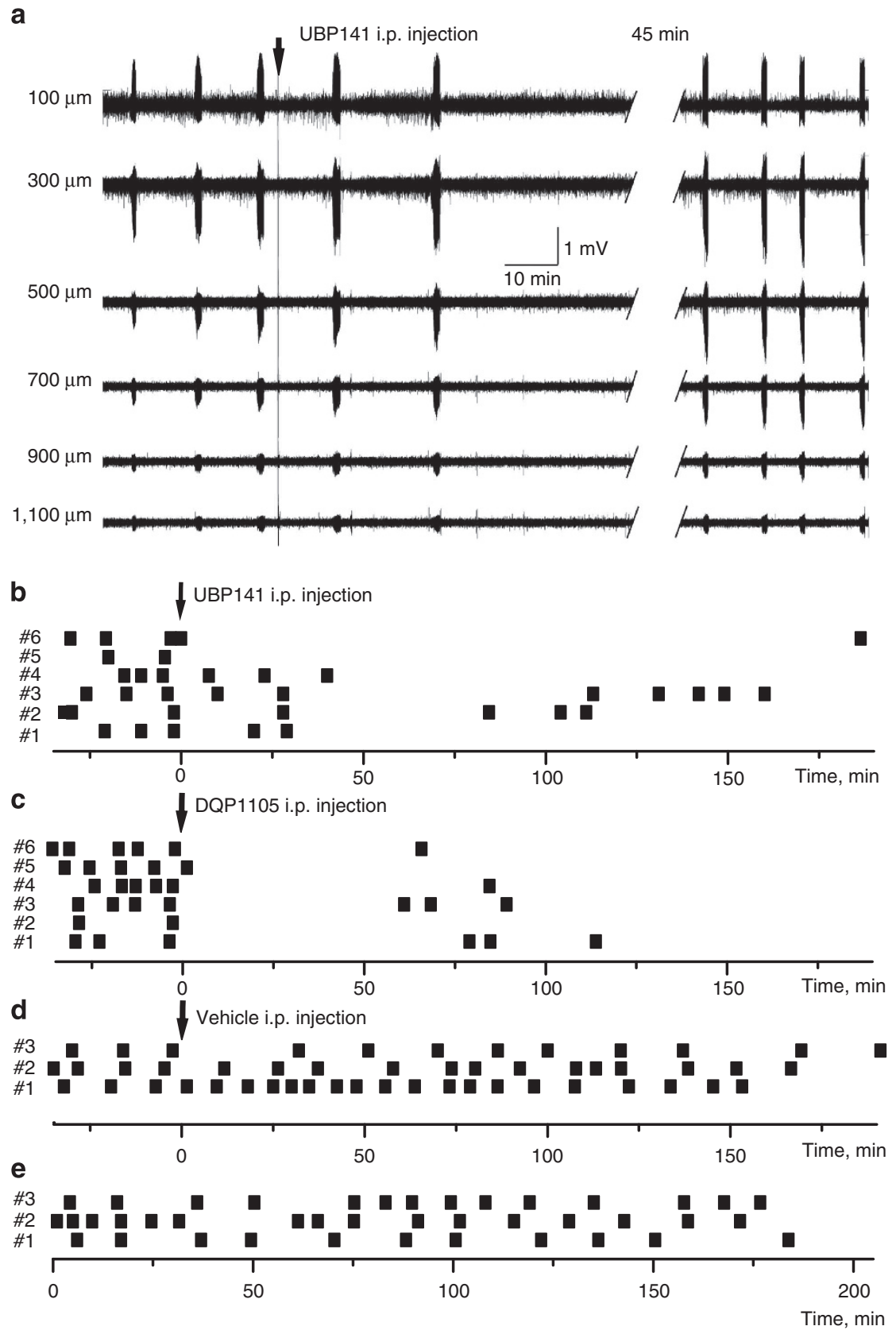

Figure 5 | Acute antiepileptic effect of i.p. administration of UBP141 or DQP1105 in vivo. (a) Intracortical EEG recordings in head-restrained P15 $\mathrm{Tsc1}^{+/-}$mouse before and after i.p. administration of UBP141 $\left(75 \mathrm{mg} \mathrm{kg}^{-1}\right.$; indicated by arrow). The upper trace corresponds to the superficial intracortical electrode placed at $100 \mu \mathrm{M}$ from the pia, channels are separated by $200 \mu \mathrm{m}$. (b-e) Time course of spontaneous seizure activity in $T s c 7^{+/-}$ mice at P14-P16 before and after i.p. administration of UBP141 ( $N=6$ mice; b), DQP1105 (28 $\mathrm{mg} \mathrm{kg}^{-1}, N=6$; c), vehicle control $(N=3$; d) and control without any treatment $(\mathrm{N}=3$; e). Individual seizures are represented by black squares. Each row (\#) represents individual experiment.

between SSCs in the granular layer of neocortex and then propagate to other layers. Interestingly, in some $T s c 1^{+/-}$mice sensory stimulation (mainly back and feet corresponding to receptive field in the recorded cortical zone) induced a seizure (Supplementary Fig. 12), indicating that sensory inputs to L4 may trigger paroxysmal recurrent network activity in the cortex. This strongly reinforces the importance of the hyper-synchronizing effects of L4 and of functional upregulation of GluN2C subunits of NMDARs.

GluN2C overexpression in human TSC postsurgical tissue. To test whether findings obtained in the animal model can be translated to human patients with TSC, we performed studies in human postsurgical tissue. Quantitative RT-PCR performed in human samples with TSC2 mutation (age at surgery ranged from 8 to 16 months; Supplementary Table 3) revealed a 20-fold increase of GluN2C mRNA compared with fetal control brains (Fig. 6a; $P<0.001$, two-tailed $t$-test) and more than 2.5 -fold increase compared with adult control brains.

Whole-cell patch-clamp recordings in brain slices from the human TSC specimens showed that sEPSCs recorded from dysplastic neurons in granular and supragranular layers (Fig. 6b) were significantly reduced and curtailed by UBP141 (Fig. 6c,d). The amplitude, total and normalized charges of sEPSC in the presence of UBP141 $(10 \mu \mathrm{M})$ were $0.78 \pm 0.04(P<0.002$, paired 

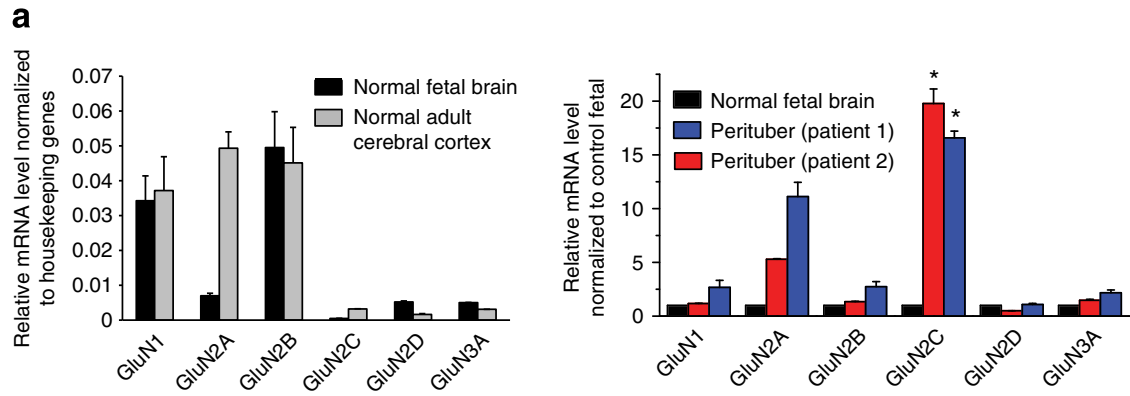

b
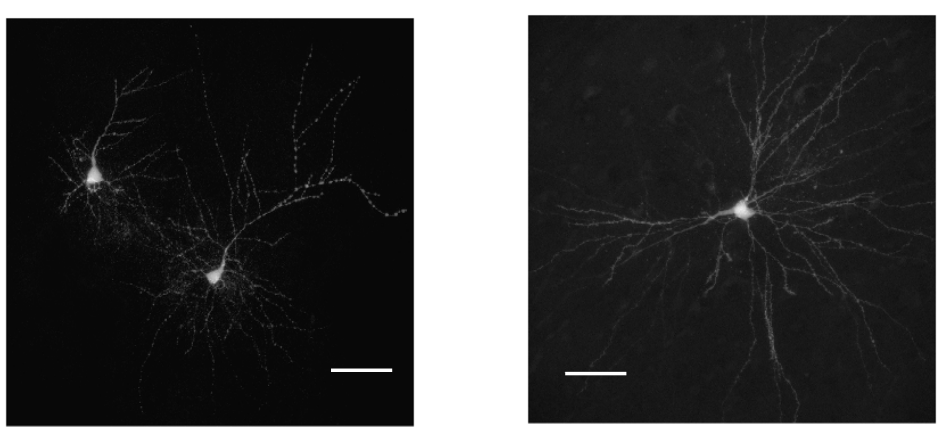

C

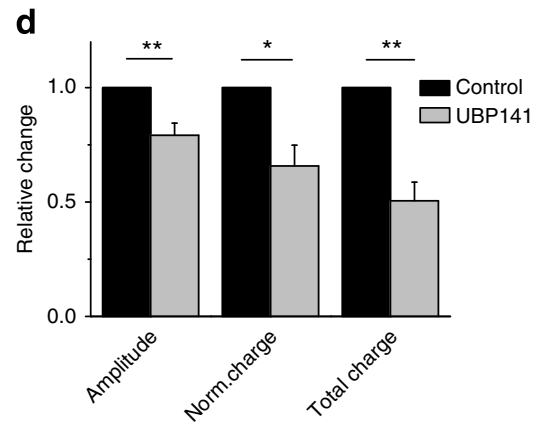

Figure 6 | Upregulation of GluN2C subunit of NMDARs in human postsurgical tissue of TSC patients. (a) Differential expression of NMDA receptor subunits in human brain tissue from normal individuals and TSC patients. Left panel: quantitative RT-PCR showing the relative expression of mRNAs encoding different NMDAR subunits in normal samples from human fetal (pool of 59 male/female Caucasian fetuses ages 20-33 weeks) and adult (pool of 10 male/female Caucasians ages 20-68 years) cerebral cortex. The error bars indicate \pm s.e.m. from two replicates of quantitative RT-PCR experiments for both sets. $\beta$-actin and GAPDH were used for normalization. Right panel: fold change expressions for each receptor in two TSC patients calculated relative to the normal fetal brain. The error bars for patient 1 indicate the \pm s.e.m. from two replicates of quantitative RT-PCR experiments. The error bars for patient 2 indicate the \pm s.e.m. from three samples. All samples were tested in duplicate. (b) Morphology of dysplastic excitatory neurons in cortical slices of human postsurgical tissue of TSC patients. Scale bar, $50 \mu \mathrm{m}$ applies to both images. (c) Grand averages of normalized (Norm.) and superimposed traces of sEPSC recorded at $-50 \mathrm{mV}$ from dysplastic neurons in cortical slices of human postsurgical tissue of three TSC patients in control (pooled data from eight neurons) and in the presence of $10 \mu \mathrm{M}$ UBP141 (pooled data from seven neurons). (d) Summary data for the effects of $10 \mu \mathrm{M}$ UBP141 on amplitudes, normalized and total charges of the sEPSC recorded from human postsurgical tissue (relative to corresponding controls, $n=7$ cells). All means \pm s.e.m., ${ }^{\star} P<0.05,{ }^{\star \star} P<0.01$; paired two-tailed $t$-tests.

two-tailed $t$-test, $n=7), 0.59 \pm 0.08(P<0.002$, paired two-tailed $t$-test, $n=7)$ and $0.68 \pm 0.09(P<0.01$, paired two-tailed $t$-test, $n=7)$, respectively, of controls. The values of $\tau_{\mathrm{w}}$ of sEPSC decay in TSC samples were $16.6 \pm 2.1 \mathrm{~ms}$ without UBP141 and $8.69 \pm 0.71 \mathrm{~ms}$ in the presence of UBP141 $(P<0.0002$, two-samples two-tailed $t$-test, $n=7$ ).

Furthermore, in two human tissue samples displaying spontaneous paroxysmal activity UBP141 $(10 \mu \mathrm{M})$ reduced spontaneous spike frequency (Supplementary Fig. 13). Therefore, in conjunction with animal model data functional upregulation of GluN2C may contribute to epilepsy associated with TSC in human patients.

Genetic polymorphisms and biochemical markers of mTOR activation have been also identified in patients with isolated focal cortical dysplasia (FCD), a common aetiology of intractable epilepsy $^{38-42}$. GluN2B and GluN2C mRNA levels have been found to be upregulated in dysplastic neurons microdissected from human FCD specimens obtained during epilepsy surgery ${ }^{26}$. Therefore, we tested whether slow UBP141-sensitive NMDARmediated component is present in dysplastic neurons from patients with FCD. Whole-cell patch-clamp recordings were performed in five human samples (age at surgery ranged from 1 to 14 years; Supplementary Table 4). As in the TSC case, sEPSCs recorded from dysplastic neurons in granular and supragranular layers (Fig. 7a) were significantly curtailed by UBP141 (Fig. 7b,c). The $\tau_{\mathrm{w}}$ values of sEPSC decay were $22.7 \pm 2.9 \mathrm{~ms}$ in control $(n=17)$ and $9.9 \pm 0.7 \mathrm{~ms}$ in the presence of UBP141 $(n=13$, $P<0.001$, two-sample, two-tailed $t$-test). The total and normalized charge of sEPSC in the presence of UBP141 $(10 \mu \mathrm{M})$ were $0.56 \pm 0.08(P<0.0003$, paired $t$-test, $n=12)$ and 


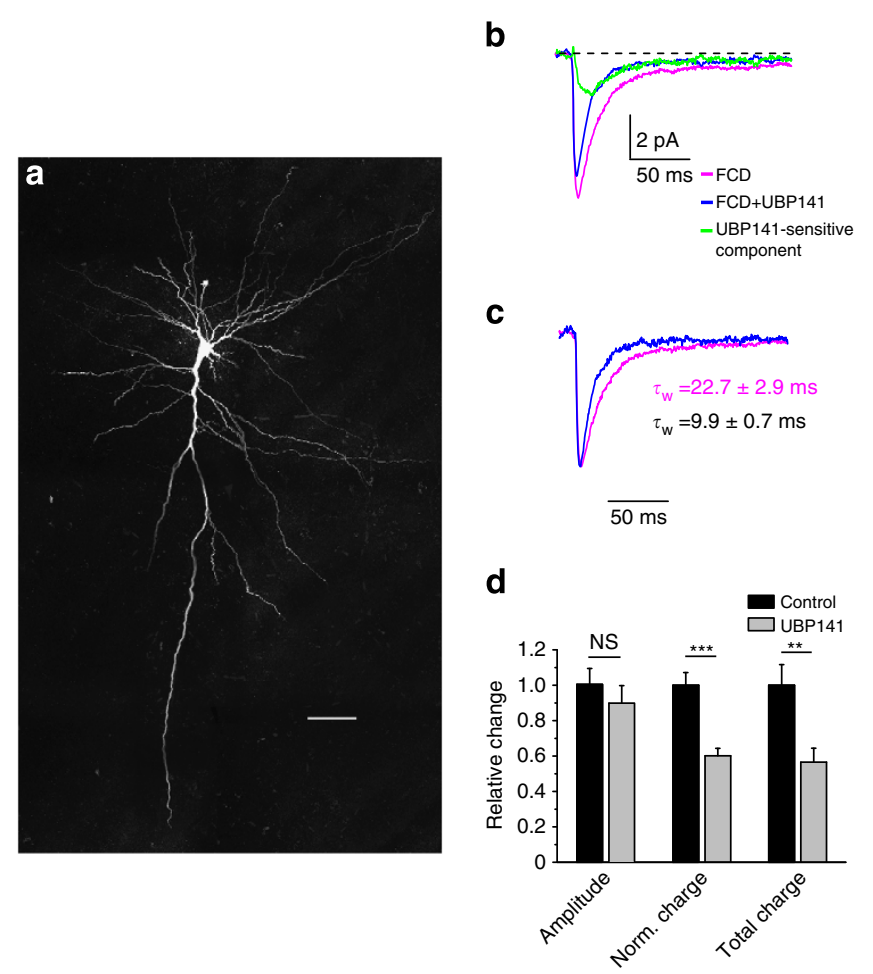

Figure 7 | Functional upregulation of GluN2C/D subunits of NMDARs in pediatric epilepsy surgery patients with focal cortical dysplasia (FCD).

(a) Morphology of dysplastic excitatory neuron in cortical slice of human postsurgical tissue of a patient with FCD. Scale bar, $50 \mu \mathrm{m}$. (b) Grand average traces of sEPSC recorded at $-50 \mathrm{mV}$ from dysplastic neurons in cortical slices of human postsurgical tissue of five patients with FCD in the absence (red, pooled data from 17 neurons) and in the presence of $10 \mu \mathrm{M}$ UBP141 (blue, pooled data from 12 neurons). (Green trace)-UBP141sensitive sEPSC component revealed by subtraction of the trace with UBP141 from the trace without UBP141. (c) Grand average traces of sEPSCs normalized (Norm.) by peak amplitudes. (d) Summary data for the effects of $10 \mu \mathrm{M}$ UBP141 on amplitudes, normalized and total charges of the sEPSC recorded from human postsurgical tissue relative to corresponding controls. All means \pm s.e.m., ${ }^{\star \star} P<0.01,{ }^{\star \star \star} P<<0.001$. Two-sample $t$-tests were performed on the pooled data for each parameter (control conditions: $n=17$ cells, with UBP141: $n=12$ cells). NS, not significant.

$0.59 \pm 0.06\left(P<2 \times 10^{-5}\right.$, paired two-tailed-test, $\left.n=12\right)$ of controls, respectively. Amplitudes of sEPSC in the presence of antagonists were not significantly different from control: $0.88 \pm 0.07$ of control $(P>0.16$, two-tailed $t$-test; Fig. $7 d)$.

Thus, although our direct results are designed for studying the mechanisms of epileptogenesis associated with TSC1 mutation, they may provide important link for other neurodevelopmental disorders with epilepsy associated with mTOR activation such as FCD.

\section{Discussion}

Our observations provide the first evidence that GluN2C NMDARs are functionally upregulated in neurons of heterozygote $T s c 1^{+/-}$mice and in human TSC patients. The upregulated expression of GluN2C receptors in neocortical L4 appear as prerequisite for seizure generation in the developing haploinsufficient $T s c 1^{+/-}$mice despite the absence of tubers and are key factors in the mechanisms of intracortical epileptogenesis. In line with this concept, GluN2C/D receptor antagonists stop seizures in vivo and in vitro. Our data with rapamycin-treated mice indicate a crucial role of mTOR signalling in increased functional expression of GluN2C NMDARs in $T s c 1^{+/-}$mice. Finally, examination of postsurgical tissue samples from TSC patients revealed that these findings could be potentially translated to human. Overall, our data suggest that GluN2Csubunit targeted therapy provides a promising novel therapeutic avenue to treat epilepsy associated with TSC.

Our in vivo recordings show that $T s c 1^{+/-}$mice exhibit a variety of seizure types (most often generalized, up to $7 \mathrm{~min}$ duration). Fast Fourier transform power spectra analysis of the EEG recordings revealed increased contribution of fast and ultrafast $(200-500 \mathrm{~Hz})$ oscillations-an important hallmark of epileptiform activity. EEG patterns in $T s c 1^{+/}-$mice resemble those seen in human epileptic seizures, and are typically associated with remarkable behavioural phenotype: freezing, facial automatisms, head tremor, straub tail and tonic-clonic seizures. Seizures in this animal model, therefore, entirely fulfil the criteria and definition of epileptic seizures proposed by the International League Against Epilepsy and the International Bureau for Epilepsy ${ }^{43}$.

However, some features and clinical phenotype in this animal model are distinctive from the human disease: in particular in mice seizures spontaneously disappear after P19, whereas in children with TSC seizures are usually highly persistent, although seizure type may change with age.

Both Tsc1 and Tsc2 heterozygous knockout mice have been intensively studied in the last years. $T s c 1^{+/-}$and $T s c 2^{+/-}$mice show social and cognitive deficits in the absence of any apparent major cerebral pathology $4,19,20$, although loss of a single copy of Tsc gene is sufficient to perturb neuronal morphology, dendritic spine structure ${ }^{22}$ and axon guidance ${ }^{44}$. Eventually, it has been proposed as a model in which haploinsufficiency for the Tsc genes leads to aberration in neuronal functioning, including changes in synaptic strength and glutamate receptor composition of excitatory synapses, resulting in impaired learning and social behaviour. Importantly, the mTOR complex 1 (mTORC1) inhibitor rapamycin improves learning and memory deficits in $T s c 2^{+/-}$mice $^{4}$, and social deficit in both $T s c 1^{+/-}$and $T s c 2^{+/-}$mice, suggesting that uncontrolled mTORC1 signalling is a core molecular mechanism involved in the behavioural abnormalities ${ }^{19,45}$.

Thus, although mice haploinsufficient for the Tsc genes have been utilized as models of autism ${ }^{4,19,20}$, occurrence of spontaneous seizures in $T s c 1^{+/-}$and $T s c 2^{+/-}$rodents have not been reported previously ${ }^{46}$. It is possible that early spontaneous epileptic activity in $T s c 1^{+/-}$heterozygous knockout mice has been overlooked because of the strictly limited age window $(<$ P19) found in our study. Interestingly, in $82.1 \%$ of epileptic TSC patients, the epilepsy begins before 3 years of life ${ }^{47}$. Thus, this early seizure appearance in $T s c 1^{+/-}$mice is consistent with the early epilepsy onset in TSC patients.

Spontaneous seizure discharges in $T s c 1^{+/}$mice involve neocortical L4 before supra- and infragranular layers, both in vivo and in vitro, indicating layer L4 as a possible origin of seizure generation. L4 is the main acceptor of sensory inputs in which SSCs act as effective integrators of powerful and persistent NMDAR-mediated recurrent excitation ${ }^{29,31}$. On the other hand, L4 neurons provide synaptic outputs to virtually all layers in a cortical column (for review, see ref. 27). We suggest that in $T s c 1^{+/-}$mice, L4 SSCs with functionally upregulated GluN2C receptors become effective 'hyperintegrators' of powerful recurrent excitation and intracortical epileptic generator. It has been hypothesized that normal brain circuits provide a template that epileptic brain uses to generate seizures ${ }^{48}$, and in particular that circuits responsible for generating seizure activity are comparable to the circuits that generate spindle bursts ${ }^{49}$. The rhythmic spike-wave discharge has been referred to as a 
perversion of the spindle oscillation ${ }^{50}$ with the circuits within the cortex amplifying the spindle activity. This hypothesis was derived primarily from CSD analysis of cortical region during spindle activity ${ }^{51}$, showing that early significant current sink occurs in cortical L4. This is in line with our own observations. Intriguingly, tactile stimulation of the $\mathrm{Tscl}^{+/-}$mouse back induced prominent seizures (Supplementary Fig. 12), indicating that seizure initiation can be triggered by conventional sensory inputs to L4.

Extensive recurrent connectivity due to upregulated GluN2C receptors in granular layer provide a source of powerful local excitation and is capable of producing activity that is selfgenerated and long-lasting. Rhythmic activity in the EEG $\gamma$-band is a near-ubiquitous feature of ongoing cortical activity and responses to sensory input, and this activity has been found selectively enhanced in epilepsy ${ }^{52}$. Combined computational and experimental approaches show that high-frequency $\gamma$ rhythms originate particularly from L4 (ref. 53). Our data show that slow decay of the GluN2C-mediated current contributes more efficiently to temporal summation at high-frequency activity. Indeed, direct paired recordings of recurrent connections between SSCs in L4 revealed increased temporal integration of excitatory input in $T s c 1^{+/-}$mice at $\gamma$-band frequency synaptic input. As a result neurons become more depolarized for a longer time window promoting neuronal hypersynchronization within L4. In concordance with patch-clamp data, i.p. injection of GluN2C/D antagonists stopped recurrent seizures in $\mathrm{Tscl}^{+/-}$mice in vivo.

Intriguingly, the impact of «second-hit» mutation on functional upregulation of slow NMDARs in neurons was negligible. Precisely, controlling the timing and location of Cre expression, we could compare the cell-autonomous effects of loss of $T s c 1$ in an otherwise unperturbed circuit or loss of both alleles in heterozygous background in contrast to the mouse models where $T s c 1$ is deleted in all forebrain neurons. The results showed that extent of slow NMDAR receptor contribution was the same irrespectively whether neurons were heterozygote or homozygote for the $T s c 1$ mutation. This indicates that monoallelic mutation in the $T s c 1$ gene is sufficient to increase the functional expression of GluN2C receptors.

Altered expression NMDAR mRNA has been reported for both human TSC and $\mathrm{FCD}^{24,26}$. In particular increased levels of GluN2B and GluN2D subunits mRNA were observed in tubers ${ }^{24}$. These data differ from present data where selective upregulation of GluN2C was observed. This discrepancy might be attributed to the difference in methods used and different age group of patients: 1-year-old patients in our study and 4-46-year-old (on average $19.5 \pm 6$ years) patients in ref. 24. However, selective upregulation of GluN2C-subunit mRNA in single microdissected dysplastic neurons (shown in ref. 24) is in a good agreement with functional upregulation of GluN2C/D subunits shown by our electrophysiological data in individual dysplastic neurons. Importantly, sEPSCs recorded from dysplastic neurons brain slices resected from patients with TSC and FCD were significantly attenuated and curtailed by GluN2C/D antagonists. Thus, given an increased expression of specific NMDAR subunits found in tissue resected from patients with various types of drug-resistant epilepsy $\mathrm{y}^{25,26,54,55}$, the proposed mechanisms of intracortical epileptogenesis and NMDAR-subunit targeted therapy may be further extended to other types of epilepsy, including FCD, the most frequent congenital lesions causing epilepsy.

It should be noted, however, that studies on postsurgery samples from TSC and FCD patients need to be interpreted with some caution. This includes our comparative findings from paediatric tissue from TSC patients ( 1-year old) with normal fetal tissue. Non-epileptic normal age-matched control tissue is not available for this human study owing to ethical issues, while comparison with age-matched epileptic tissue with different aetiology may lead to misinterpretation and wrong conclusions. Our results should be replicated in other clinical studies with larger cohorts and rigorous age correlation analysis.

The failure of general NMDAR antagonists to treat epilepsy in preclinical studies ${ }^{56}$ may result from the need to restrict the NMDARs blockade to the specific subunits involved in epileptogenesis in TSC leaving intact pro-survival GluN2Amediated signalling involved in normal development, plasticity, learning and memory ${ }^{57}$. Administration of non-specific NMDAR antagonists alters $\gamma$ rhythms and can induce cognitive as well as psychosis-like symptoms in humans ${ }^{58}$. In FS parvalbuminpositive interneurons, NMDARs have a critical role for expression of normal $\gamma$ rhythms and specific cognitive behaviours ${ }^{59}$. The disruption of NMDAR signalling specifically in parvalbuminpositive interneurons may lead to neural network dysfunction that could underlie these symptoms. The NMDARs present in FS interneurons, both in $T s c 1^{w t}$ and $T s c 1^{+/-}$mice, were not affected by selective GluN2B or GluN2C/D antagonists in our experiments. This suggests that treatment with these compounds does not alter inhibition provided by FS interneurons. Furthermore, power of $\gamma$ oscillations of basal activity recorded in $T s c 1^{+/}$mice in vivo was not affected by UBP141.

Abnormal signalling in the mTOR pathway is known to be critical for the pathophysiology of epilepsy and other neurological features of TSC, and other focal cortical malformations. In animal models of TSC and cortical dysplasia, hyperactivation of the mTOR pathway promotes epileptogenesis and neuropathological abnormalities, and the mTOR inhibitor, rapamycin, prevents epilepsy and associated cellular and molecular phenotypes ${ }^{2-4,60}$ Altered mTOR pathway signalling was found in TSC, FCD, hemimegalencephaly and ganglioglioma brain tissue specimens ${ }^{38,40,61-63}$. The term 'TORopathies' was recently coined to define a continuum of neurological disorders characterized by altered cortical architecture, abnormal neuronal morphology and intractable seizures as a consequence of abnormal mTOR signalling ${ }^{63,64}$. There is strong evidence supporting a role for mTORC1 in regulating the translation of a number of proteins necessary for synaptic plasticity ${ }^{65}$, and there is a possibility that $\mathrm{mTORC1}$ also regulates translation of GluN2C. Our findings obtained from TSC animal model may, therefore, also have implications for other conditions in which mTORC1 is hyperactive, ranging from Fragile $\mathrm{X}$ syndrome to autism spectrum disorders ${ }^{66}$.

\section{Methods}

Animals. Breeding and experimental procedures were carried out in accordance with European guidelines for animal research and in accordance with Institut National de la Santé et de la Recherche Medicale guidelines for animal care in research, and were approved by the local ethics committee (Comité d'éthique en expérimentation animale de Marseille (C2EA-14)). Heterozygote Tsc1 ${ }^{\text {wt } / m u t}$ $\left(T s c 1^{+/-}\right.$, National Cancer Institute (NCI, USA)) male mice were kindly provided by Dr A. Bordey (Yale University, USA), and Tsc1 ${ }^{f l x / f l x}$ were obtained from Jackson Laboratories. The genetic background of Tsc1 $1^{\text {wt } / m u t}$ mice was B6;129S4 and Tsc1 $1^{f x /}$ flx mice were from a mixed background (C57BL/6J, BALB/cJ, or 129/SvJae mice). These lines of mice were generated by David J. Kwiatkowski (Brigham and Women's Hospital, Harvard Medical School, Cambridge, MA, USA). Inbred C57BL/6J wild-type $\left(T s c 1^{+/+}\right)$females were from Janvier Labs (France). Mice were housed in ventilated, light-tight, sound-isolated chambers under standard 12:12 light/dark cycle (light on at 07.00 PM and light off at 07.00 AM) with food and water available ad libitum. Genotyping of pups issued from breading of C57BL/6 $T s c 1^{+/-}$males and C57BL/6J Tsc1 ${ }^{+1+}$ females mice was performed on tail tissue samples at postnatal days P11-P12. The study was conducted in $\mathrm{Tscl}^{+/-}$and $\mathrm{Tsc1} \mathrm{1}^{+/+}$mice of both sexes issued from the same litters at P9-P33.

In utero electroporation. Timed pregnant female mice at the E16 (E0 is counted as the morning on which the vaginal plug is detected) were anaesthetized with a mixture of ketamine $\left(10 \mathrm{mg} \mathrm{kg}^{-1}\right) / x y l a z i n e ~\left(100 \mathrm{mg} \mathrm{kg}^{-1}\right)$. The uterine horns were exposed, and a lateral ventricle of each embryo was injected using pulled glass 
capillaries and a microinjector (Picospritzer II; General Valve) with Fast Green ( $2 \mathrm{mg} \mathrm{ml}^{-1}$; Sigma, USA) combined with the following DNA constructs: $0.5 \mathrm{mg} \mathrm{ml}^{-1}$ pCAG-mRFP either alone or with $1.5 \mathrm{mg} \mathrm{ml}^{-1}$ pCAG-Cre:GFP. Plasmids were further electroporated by discharging a $4,000-\mathrm{mF}$ capacitor charged to $35 \mathrm{~V}$ with a BTX ECM 830 electroporator (BTX Harvard Apparatus). The voltage was discharged in five electrical pulses at $950-\mathrm{ms}$ intervals via $5-\mathrm{mm}$ electrodes placed on the head of the embryo across the uterine wall.

Quantitative RT-PCR. Total RNA was isolated from mouse cerebral cortex and human brain tissue using RNeasy-Plus Mini Kit according to the manufacturer's protocol (Qiagen). cDNA was synthesized using the Quantitect Reverse Transcription Kit, according to the manufacturer's protocol (Qiagen). Quantitative real-time RT-PCR experiments were performed using oligonucleotides specific for mouse hypoxanthine phosphoribosyltransferase 1 and cyclophilin-A; human $\beta$-actin and GAPDH, and mouse and human NMDAR subunits genes (oligonucleotide sequences are shown in Supplementary Table 5). Amplification was done using SYBR-Green and Chemistry (Roche Diagnostics) and Roche amplification technology (Light Cycler 480). All experiments were performed in duplicate or triplicate. To avoid any bias, all samples were blinded before analysis. Only after the final samples were analysed and described, the samples were genotyped. Human fetal brain and adult cerebral cortex mRNAs, used as controls, were purchased from BD Biosciences Clontech (Palo Alto, CA, USA).

Animal slice preparation. $T s c 1^{+/+}$and $T s c 1^{+/-}$mice (P14-P16) were anaesthetized with ether and killed by decapitation in agreement with the European Directive 86/609/EEC requirements.

The brain was rapidly removed and placed in an oxygenated ice-cold saline buffer. Transverse $300-\mu \mathrm{m}$-thick coronal slices were cut using a vibratome (Leica VT1000S; Leica Microsystems Inc., Deerfield, IL, USA) in ice-cold protecting solution oxygenated with $95 \% \mathrm{O}_{2}$ and $5 \%$ of $\mathrm{CO}_{2}$. Before recording, slices were incubated in an artificial cerebrospinal fluid (ACSF-1) solution containing (in $\mathrm{mM}): 125 \mathrm{NaCl}, 3.5 \mathrm{KCl}, 1 \mathrm{CaCl}_{2}, 2 \mathrm{MgCl}_{2}, 1.25 \mathrm{NaH}_{2} \mathrm{PO}_{4}, 26 \mathrm{NaHCO}_{3}$ and 10 glucose, equilibrated at $\mathrm{pH} 7.3$ with $95 \% \mathrm{O}_{2}$ and $5 \% \mathrm{CO}_{2}$ at room temperature $\left(22-25^{\circ} \mathrm{C}\right)$ for at least $1 \mathrm{~h}$ to allow recovery. For the recordings, we used ACSF of the same composition but with $2 \mathrm{mM}$ of $\mathrm{CaCl}_{2}$ and $1 \mathrm{mM}$ of $\mathrm{MgCl}_{2}$ (ASCF-2).

Immunostaining procedures. For immunostaining, P14-P16 mouse brains were perfused with Anti-genfix solution and sliced at $100 \mu \mathrm{m}$ on a vibratome (Microm). Slices were blocked at room temperature for $1 \mathrm{~h}$ with $5 \%$ normal goat serum (NGS) and $0.3 \%$ Triton X-100 in phosphate-buffered saline (PBS) and incubated overnight at $37^{\circ} \mathrm{C}$ with the upper cortical layer marker CDP/CUX1 (M-222 Santa Cruz Biotechnology; 1/200).

Human cortical slice preparation. After surgical resection, the cortical tissue was placed within $30 \mathrm{~s}$ in ice-cold oxygenated protecting solution that contained in (mM): 110 choline chloride, $26 \mathrm{NaHCO}_{3}, 10$ D-glucose, 11.6 sodium ascorbate, $7 \mathrm{MgCl}_{2}, 3.1$ sodium pyruvate, $2.5 \mathrm{KCl}, 1.25 \mathrm{NaH}_{2} \mathrm{PO}_{4}$ and $0.5 \mathrm{CaCl}_{2}, 300 \mathrm{mOsm}$ and transported to the neurophysiology laboratory, within $<5 \mathrm{~min}$. Cortical slices $(400-900 \mu \mathrm{m})$ were prepared in the same solution, and were then transferred to holding chambers in which they were stored at room temperature $\left(20-22^{\circ} \mathrm{C}\right)$ in ACSF-1. Recordings were performed in ACSF-2.

Electrophysiological recordings from brain slices. Slices were transferred to the recording chamber and perfused with oxygenated recording ACSF-2 at $3 \mathrm{ml} \mathrm{min}{ }^{-1}$. Neurons were visualized using infrared differential interference contrast microscopy. Whole-cell patch-clamp recordings were performed at room temperature by using either an EPC-9 amplifier and Patch Master software (HEKA Elektronik, Germany) or Multiclamp 700B amplifier (Molecular Devices, Sunnyvale, CA, USA) and custom-made software based on IgorPro and filtered at 3-10 kHz. Patch pipettes were pulled from borosilicate glass capillaries (World Precision Instruments, Sarasota, USA) and had resistances of 4-6.5 M $\Omega$ when filled with the internal solution of the following composition (in $\mathrm{mM}$ ): $130 \mathrm{~K}$-gluconate, $10 \mathrm{Na}$-gluconate, $4 \mathrm{NaCl}, 4 \mathrm{MgATP}, 4$ phosphocreatine, 10 HEPES and $0.3 \mathrm{GTP}$ (pH 7.3 with KOH). Biocytin (final concentration $0.3-0.5 \%$ ) was added to the pipette solution to label the neurons from which recordings were obtained.

The series resistance estimated from the amplitude of the initial capacitive transient in response to a 5-mV pulse was 8-24 M 2 . It was not compensated and was monitored during each experiment. Experiments were terminated if the series resistance changed by $>15 \%$. Spontaneous EPSCs were recorded for $15 \mathrm{~min}$ at $-80 \mathrm{mV}$ (the reversal potential for GABAergic currents) and at $-50 \mathrm{mV}$ (potential at which the block of NMDAR by magnesium is largely relieved). All recordings were made in ACSF-2 without any proepileptic pharmacological drug. To minimize potential sampling bias, the pups from at least three deliveries for each condition were studied.

Analysis and statistics of in vitro data. The Mini Analysis 6.0 .3 software (Synaptosoft Inc., Decatur, GA, USA) was used to analyse the parameters of synaptic events. The threshold amplitude for detecting EPSCs was set at twice the baseline noise (root mean square), and the EPSCs detected by the software were visually inspected to minimize errors. Events that did not show a typical synaptic waveform were rejected manually.

For analysis, only events that did not show any signs of multiple peaks (that is, contamination of rise or decay phases by subsequent events) were selected for subsequent analysis of the kinetics and for exponential fitting.

Averaged traces of sEPSC or mEPSC and cumulative probabilities of amplitude and frequency for all cells were obtained using (Mini Analysis; Synaptosoft) software and further analysed with Origin software (MicroCal, Northampton, MA, USA).

For each neuron, original traces from individual experiments were aligned based on the starts of their rising phases and averaged. These averaged traces from individual experiments were averaged to form grand average traces.

To quantify the current decay kinetics, we measured charge transfer of sEPSC normalized by the peak amplitude. A larger charge transfer corresponds to slower decay kinetics and vice versa. Normalized charge transfer was calculated by the integrating the area under the current waveform in the interval of time between peak of sEPSC and $300 \mathrm{~ms}$ after the peak for each individual cell (Supplementary Fig. 14).

In addition, to compare decay times between genotypes weighted time constant of sEPSC or mEPSC decay $\left(\tau_{\mathrm{w}}\right)$ were calculated using the formula (equation 1):

$$
\tau_{\mathrm{W}}=\left(I_{\text {fast }} /\left(I_{\text {fast }}+I_{\text {slow }}\right)\right) * \tau_{\text {fast }}+\left(I_{\text {slow }} /\left(I_{\text {slow }}+I_{\text {fast }}\right)\right) * \tau_{\text {slow }},
$$

where $I$ is the amplitude of the fast or slow component, and $\tau$ is the respective decay time constant.

Data are expressed as mean \pm s.e.m. All comparisons were two-tailed $t$-tests or one-way ANOVA with post hoc Fisher's Least Significant Difference multiple comparison. Statistical significance for cumulative probabilities was estimated using Kolmogorov-Smirnov and Mann-Whitney tests. From three to nine animals were used for each data set.

Immunocytochemistry. After the recording session, to visualize and identify the recorded neurons, we visualized the biocytin injected during whole-cell recordings. After $24 \mathrm{~h}$ in paraformaldehyde $(3 \%)$ at $4{ }^{\circ} \mathrm{C}$, the sections were rinsed in PBS and pre-incubated for $1 \mathrm{~h}$ in $0.3 \%$ Triton X-100 (Abcys) in PBS with 5\% NGS at room temperature. Slices were then incubated in Streptavidin-Cy3 (1:500) in PBS Triton $\mathrm{X}-100(0.3 \%)$ and NGS (5\%) during $12 \mathrm{~h}$ at $4{ }^{\circ} \mathrm{C}$. After thorough rinsing, slices were mounted in fluoromount and coverslipped.

In vivo recordings and data analysis. Experiments were performed on postnatal days P9-P33 of inbred C57BL/6J strain of both sexes of $T s c 1^{+/-}$and $T s c 1^{w t}$ mice from the same litters issued from breading of $T s c 1^{w t}$ females and $T s c 1^{+/-}$males. The available littermates mice were randomly picked out of the cage and genotyped after experiments. Surgery was performed under isoflurane anaesthesia. In brief, the skull of the animal was cleaned of skin and periosteum. The skull was covered by glue and dental cement except for a $4-9 \mathrm{~mm}^{2}$ window above the somatosensory cortex from one or two hemispheres. Two plastic bars were fixed to the nasal and occipital bones of the pups head by dental cement. After surgery, animals were warmed and were left for an hour for recovery from anaesthesia. During recordings, the head was fixed to the frame of the stereotaxic apparatus by attached bars; animals were surrounded by a cotton nest and heated via a thermal pad $\left(36.6-37.7^{\circ} \mathrm{C}\right)$. A silver chloride reference electrode was placed in the cerebellum or visual cortex.

EEG recordings were performed in non-anaesthetized head-restrained $T s c 1^{+/-}$and control $T s c 1^{w t}$ mice. Sixteen site-linear silicon probe $(100 \mu \mathrm{m}$ separation distance between recording sites, Neuronexus Technologies, MI, USA) was placed into the somatosensory cortex using the Paxinos and Franklin atlas (2001) at coordinates: anterior-posterior $=2-2.5 \mathrm{~mm}$, mediolateral $=2-3 \mathrm{~mm}$ from Bregma, $1.2-1.5 \mathrm{~mm}$ depth, to trace the columnar activity at all layers and CA1 zone of the hippocampus. Signals were amplified $(\times 100)$ and filtered at $3 \mathrm{kHz}$ using a 16-channel amplifier (A-M systems, Inc.), digitized at $10 \mathrm{kHz}$ and saved to hard disk of PC using Axoscope software (Molecular Devices). Recordings were analysed offline using Clampfit and MATLAB software (The Mathworks, Natick, MA, USA).

After the recordings, position of silicone probe was verified visually by DiI staining of the electrode in $100 \mu \mathrm{m}$ coronal sections from fixed brain. The actions of UBP141 and DQP1105 were studied in vivo animal model of TSC in keeping with recent guidelines for preclinical candidate drug evaluation ${ }^{67}$.

We considered that multiunit activity occurred in epileptic discharges if they appeared in a group of multiple spikes whose amplitude exceeded at least twice the background activity within a period lasting for at least $20 \mathrm{~s}$. The first and last spikes of each discharge were used to define its onset and termination, respectively. For each discharge, amplitude was defined as the amplitude of the largest spike of the discharge. During EEG recordings, animals were monitored visually to determine behavioural correlates of each electrographic epileptic discharge.

For EEG data analysis, raw data were preprocessed using a custom-developed suite of programs in the MATLAB analysis environment. The wide-band signal was downsampled to $1,000 \mathrm{~Hz}$ and used for local field potential (LFP) signal. Positive polarity is graphed as up throughout the whole manuscript. 
LFPs were analysed by custom-written, MATLAB-based programs.

Approximate anatomical location of each recording site was estimated by physical depth within the brain and corresponding age-matched histological assessment of respective layers depth.

Negative epileptic events were detected by the following steps: (1) the LFP signal was band-pass filtered $(5-100 \mathrm{~Hz})$; (2) the times of negative troughs with amplitude $>5$ s.d. from baseline level were detected from filtered signal; and (3) aligned by the times of detected negative epileptic events; the LFP segments from all channels were taken. Note that the length of the segment is $200 \mathrm{~ms}$ with moment of negative trough at 0 .

CSD analysis across cortical depth was used to eliminate volume conduction and localize synaptic currents. CSD was computed for each recording site according to differential scheme for second derivative and smoothed with a triangular kernel of length 3 .

Preliminary screen for UBP141 and DQP1105 efficacy in vivo. Saline solution

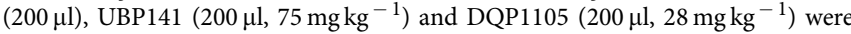
injected i.p. Using in silico prediction of blood-brain barrier permeation, we estimated expected concentration of compounds in the brain. The decimal logarithm of brain to blood concentration ratio $(\log B B)$ and drug concentration relationship in the brain and blood (plasma) tissues were reconstructed using the Clark's equation ${ }^{68}$ to assess the $\log B \mathrm{~B}$ value for the UBP141 and DQP1105 compounds (equation (2)):

$$
\log \mathrm{BB}=0.152 \mathrm{Clog} \mathrm{P}-0.0148 \mathrm{PSA}+0.139,
$$

where $C \log \mathrm{P}$ is a calculated octanol-water partitioning coefficient, and PSA is a polar surface area (Supplementary Table 6 and Supplementary Fig. 15).

Both parameters were determined from molecular fields using the VolSurf + molecular modelling software programme (Molecular Discovery Ltd, Perugia, Italy $)^{69}$. According to this model the pharmacodynamic distribution of the drugs in the brain after i.p. injection was assessed at least as $6.7 \%$ and $15 \%$ of the initial concentration in blood for UBP141 and DQP1105, respectively.

On the other hand, according to ref. 70, the free-drug concentration in the peritoneal cavity is described as its exponential decrease over distance $(x)$ from the serosal surface (peritoneum) since the drug diffuses down the concentration gradient into the bloodstream (Supplementary Fig. 16). At characteristic diffusion length, $\times 0$ (average distance travelled by drug molecules), the concentration difference between tissue and blood is decreased to $37 \%$ of its maximum value.

Therefore, considering the decline of drug concentration at $\times 0$ due to peritoneal transport, and the $\log \mathrm{BB}$ values the distribution of DQP1105 in the brain after i.p. injection can be assessed. Assuming DQP1105 concentration in injected solution $(2.5 \mathrm{mM})$, volume of injection $(200 \mu \mathrm{l})$ and total circulating blood volume (of $\sim 1,000 \mu \mathrm{l}$ ), concentration in brain will be around $25 \mu \mathrm{M}$ that is entirely within the selectivity range for GluN2C/D (Supplementary Table 1). Similar estimation for UBP141 is complicated by the fact that effect of UBP141 (in contrast to DQP1105) was delayed for $40 \mathrm{~min}$ after i.p. injection, and unknown pharmacokinetic profile could significantly influence the estimation.

Preliminary screen for efficacy of UBP141 and DQP1105 was also performed directly to detect threshold for antiepileptic activity. In 12 animals tested, i.p. injection of UBP141 with dosages of 19 or $57 \mathrm{mg} \mathrm{kg}^{-1}$ did not produce significant antiepileptic effects. In three animals tested, i.p. injection of DQP1105 with a dosage of $14 \mathrm{mg} \mathrm{kg}^{-1}$ was also ineffective. A dosage of $75 \mathrm{mg} \mathrm{kg}^{-1}$ for UBP141 and $28 \mathrm{mg} \mathrm{kg}^{-1}$ for DQP1105 were the lowest effective doses identified by testing increasing doses of these compounds.

Multisite extracellular recordings from brain slices. Multisite extracellular recordings of spontaneous activity in slices were performed at $30-32{ }^{\circ} \mathrm{C}$ using Multi-electrode array (MEA) made up of 60 planar microelectrodes (TiN/SiN $30 \mu \mathrm{m}$ electrode diameter, $200 \mu \mathrm{m}$ pitch) arranged over an $8 \times 8$ square grid (Multi Channel Systems (MCS), Reutlingen, Germany). Slices were maintained in dishes and perfused with oxygenated recording ACSF-2. The spontaneous activity was monitored and recorded for 30-120 min, starting 15-20 min after setting slice in the recording chamber at a stable level of activity. After 1,200 $\times$ amplification (MCS MEA 1060), signals were sampled at $10 \mathrm{kHz}$ using the MCS data acquisition card controlled by the MCS MCRack software. Data were analysed offline by using custom software tools specifically developed in MATLAB

Chemicals. All drugs were prepared as concentrated stock solutions (10-100 mM), stored frozen and then thawed and diluted in ACSF-2 immediately before use Ro-25697 ( $\alpha \mathrm{R}, \beta S$ )- $\alpha$-(4-hydroxyphenyl)- $\beta$-methyl-4-(phenylmethyl)-1-piperidinepropanol maleate, and DQP1105 5-(4-bromophenyl)-3-(1,2-dihydro-6methyl-2-oxo-4-phenyl-3-quinolinyl)-4,5-dihydro-g-oxo-1H-pyrazole-1-butanoic acid were purchased from Tocris Biosciences (Bristol, UK). UBP $141\left(2 \mathrm{R}^{\star}, 3 \mathrm{~S}^{*}\right)-1$ (phenanthrenyl-3-carbonyl)piperazine-2,3-dicarboxylic acid was purchased from Abcam Biochemicals (UK). All other chemicals used for electrophysiology were from Sigma.

Treatment with rapamycin. Rapamycin (ready-made solution, Sigma-Aldrich, USA, $2.5 \mathrm{mg} \mathrm{ml}^{-1}$ in DMSO $(2.74 \mathrm{mM})$ ) was diluted with saline to a final concentration $0.125 \mathrm{mg} \mathrm{ml}^{-1}$ immediately before use. The mice received single dose of rapamycin $\left(3 \mathrm{mg} \mathrm{kg}^{-1}\right)$ by i.p. injection $(100 \mu \mathrm{l})$ or were injected by the equal volume of vehicle once daily for 8 consecutive days. The electrophysiological recordings were performed $24 \mathrm{~h}$ after the last administration.

Human subjects. Cortical tissue samples were obtained from three TSC epilepsy patients undergoing surgery at the Departments of Pediatric Neurosurgery of Rothschild Foundation (Paris) and Hopital La Timone (Marseille). Informed consent for the use of postsurgical tissue for research purposes was obtained with protocols approved by the Rothschild Foundation and Hopital La Timone review boards. Personal data are stored into a specific database, which is declared to the $\mathrm{La}$ Commission nationale de l'informatique et des libertés (CNIL). Bioethical declaration procedure, related to conservation and preparation of human body elements for scientific aims, was achieved during 2011 as referred to the 'Collection NeurochirEpilepsie` under file number DC-2011-1378, and has been approved by Le Comite de Protection des Personnes Ile de France II and Ministère de l'enseignement supérieur et de la recherche.

The main clinical and neuropathological characteristics of the study population are summarized in Supplementary Tables 3 and 4. TSC and FCD specimens were collected from patients who underwent surgery for medically intractable epilepsy. All patients were clinically diagnosed with TSC and FCD and presented a history of epilepsy.

\section{References}

1. Curatolo, P. in Sclerosis Complex From Basic Science To Clinical Phenotypes (I.C.N. AssociationMac Keith Press, 2003).

2. Zeng, L. H., Xu, L., Gutmann, D. H. \& Wong, M. Rapamycin prevents epilepsy in a mouse model of tuberous sclerosis complex. Ann. Neurol. 63, 444-453 (2008).

3. Meikle, L. et al. Response of a neuronal model of tuberous sclerosis to mammalian target of rapamycin (mTOR) inhibitors: effects on mTORC1 and Akt signaling lead to improved survival and function. J. Neurosci. 28, 5422-5432 (2008).

4. Ehninger, D. et al. Reversal of learning deficits in a Tsc2 $+/$ - mouse model of tuberous sclerosis. Nat. Med. 14, 843-848 (2008).

5. Curatolo, P., Bombardieri, R. \& Jozwiak, S. Tuberous sclerosis. Lancet 372, 657-668 (2008).

6. Henske, E. P. et al. Loss of tuberin in both subependymal giant cell astrocytomas and angiomyolipomas supports a two-hit model for the pathogenesis of tuberous sclerosis tumors. Am. J. Pathol. 151, 1639-1647 (1997).

7. Qin, W. et al. Analysis of TSC cortical tubers by deep sequencing of TSC1, TSC2 and KRAS demonstrates that small second-hit mutations in these genes are rare events. Brain Pathol. 20, 1096-1105 (2010).

8. Crino, P. B., Aronica, E., Baltuch, G. \& Nathanson, K. L. Biallelic TSC gene inactivation in tuberous sclerosis complex. Neurology 74, 1716-1723 (2010).

9. Curatolo, P., Bombardieri, R. \& Cerminara, C. Current management for epilepsy in tuberous sclerosis complex. Curr. Opin. Neurol. 19, 119-123 (2006).

10. Jansen, F. E., van Huffelen, A. C., Algra, A. \& van Nieuwenhuizen, O. Epilepsy surgery in tuberous sclerosis: a systematic review. Epilepsia 48, 1477-1484 (2007).

11. Wu, W. E. et al. Brain MR spectroscopic abnormalities in 'MRI-negative' tuberous sclerosis complex patients. Epilepsy Behav. 27, 319-325 (2013).

12. Major, P. et al. Are cortical tubers epileptogenic? Evidence from electrocorticography. Epilepsia 50, 147-154 (2009).

13. Kaufmann, R., Kornreich, L. \& Goldberg-Stern, H. Unusual clinical presentation of tuberless tuberous sclerosis complex. J. Child. Neurol. 24, 361-364 (2009).

14. Cusmai, R., Chiron, C., Curatolo, P., Dulac, O. \& Tran-Dinh, S. Topographic comparative study of magnetic resonance imaging and electroencephalography in 34 children with tuberous sclerosis. Epilepsia 31, 747-755 (1990).

15. Feliciano, D. M., Su, T., Lopez, J., Platel, J. C. \& Bordey, A. Single-cell Tsc1 knockout during corticogenesis generates tuber-like lesions and reduces seizure threshold in mice. J. Clin. Invest. 121, 1596-1607 (2011).

16. Meikle, L. et al. A mouse model of tuberous sclerosis: neuronal loss of Tsc1 causes dysplastic and ectopic neurons, reduced myelination, seizure activity, and limited survival. J. Neurosci. 27, 5546-5558 (2007).

17. Uhlmann, E. J. et al. Astrocyte-specific TSC1 conditional knockout mice exhibit abnormal neuronal organization and seizures. Ann. Neurol. 52, 285-296 (2002).

18. Way, S. W. et al. Loss of Tsc2 in radial glia models the brain pathology of tuberous sclerosis complex in the mouse. Hum. Mol. Genet. 18, 1252-1265 (2009).

19. Chevere-Torres, I., Maki, J. M., Santini, E. \& Klann, E. Impaired social interactions and motor learning skills in tuberous sclerosis complex model mice expressing a dominant/negative form of tuberin. Neurobiol. Dis. 45, 156-164 (2011). 
20. Goorden, S. M., van Woerden, G. M., van der Weerd, L., Cheadle, J. P. \& Elgersma, Y. Cognitive deficits in Tscl $+/$ - mice in the absence of cerebral lesions and seizures. Ann. Neurol. 62, 648-655 (2007).

21. Bateup, H. S. et al. Excitatory/Inhibitory synaptic imbalance leads to hippocampal hyperexcitability in mouse models of tuberous sclerosis. Neuron 78, 510-522 (2013).

22. Tavazoie, S. F., Alvarez, V. A., Ridenour, D. A., Kwiatkowski, D. J. \& Sabatini, B. L. Regulation of neuronal morphology and function by the tumor suppressors Tsc1 and Tsc2. Nat. Neurosci. 8, 1727-1734 (2005).

23. Zilles, K., Qu, M. S., Kohling, R. \& Speckmann, E. J. Ionotropic glutamate and GABA receptors in human epileptic neocortical tissue: quantitative in vitro receptor autoradiography. Neuroscience 94, 1051-1061 (1999).

24. White, R. et al. Selective alterations in glutamate and GABA receptor subunit mRNA expression in dysplastic neurons and giant cells of cortical tubers. Ann. Neurol. 49, 67-78 (2001).

25. Talos, D. M., Kwiatkowski, D. J., Cordero, K., Black, P. M. \& Jensen, F. E. Cell-specific alterations of glutamate receptor expression in tuberous sclerosis complex cortical tubers. Ann. Neurol. 63, 454-465 (2008).

26. Crino, P. B., Duhaime, A. C., Baltuch, G. \& White, R. Differential expression of glutamate and GABA-A receptor subunit mRNA in cortical dysplasia. Neurology 56, 906-913 (2001).

27. Feldmeyer, D. Excitatory neuronal connectivity in the barrel cortex. Front. Neuroanat. 6, 24 (2012).

28. Beierlein, M., Fall, C. P., Rinzel, J. \& Yuste, R. Thalamocortical bursts trigger recurrent activity in neocortical networks: layer 4 as a frequency-dependent gate. J. Neurosci. 22, 9885-9894 (2002).

29. Feldmeyer, D., Egger, V., Lubke, J. \& Sakmann, B. Reliable synaptic connections between pairs of excitatory layer 4 neurones within a single 'barrel' of developing rat somatosensory cortex. J. Physiol. 521(Pt 1): 169-190 (1999).

30. Binshtok, A. M., Fleidervish, I. A., Sprengel, R. \& Gutnick, M. J. NMDA receptors in layer 4 spiny stellate cells of the mouse barrel cortex contain the NR2C subunit. J. Neurosci. 26, 708-715 (2006).

31. Fleidervish, I. A., Binshtok, A. M. \& Gutnick, M. J. Functionally distinct NMDA receptors mediate horizontal connectivity within layer 4 of mouse barrel cortex. Neuron 21, 1055-1065 (1998).

32. Lefort, S., Tomm, C., Floyd Sarria, J. C. \& Petersen, C. C. The excitatory neuronal network of the $\mathrm{C} 2$ barrel column in mouse primary somatosensory cortex. Neuron 61, 301-316 (2009).

33. Monyer, H., Burnashev, N., Laurie, D. J., Sakmann, B. \& Seeburg, P. H. Developmental and regional expression in the rat brain and functional properties of four NMDA receptors. Neuron 12, 529-540 (1994).

34. Costa, B. M. et al. N-methyl-D-aspartate (NMDA) receptor NR2 subunit selectivity of a series of novel piperazine-2,3-dicarboxylate derivatives: preferential blockade of extrasynaptic NMDA receptors in the rat hippocampal CA3-CA1 synapse. J. Pharmacol. Exp. Ther. 331, 618-626 (2009).

35. Acker, T. M. et al. Mechanism for noncompetitive inhibition by novel GluN2C/D N-methyl-D-aspartate receptor subunit-selective modulators. Mol. Pharmacol. 80, 782-795 (2011).

36. Ichikawa, T. et al. A case of solitary subependymal giant cell astrocytoma: two somatic hits of TSC2 in the tumor, without evidence of somatic mosaicism. J. Mol. Diagn. 7, 544-549 (2005).

37. Hakami, T. et al. NMDA receptor hypofunction leads to generalized and persistent aberrant gamma oscillations independent of hyperlocomotion and the state of consciousness. PLoS ONE 4, e6755 (2009).

38. Baybis, M. et al. mTOR cascade activation distinguishes tubers from focal cortical dysplasia. Ann. Neurol. 56, 478-487 (2004).

39. Becker, A. J. et al. Focal cortical dysplasia of Taylor's balloon cell type: mutational analysis of the TSC1 gene indicates a pathogenic relationship to tuberous sclerosis. Ann. Neurol. 52, 29-37 (2002).

40. Ljungberg, M. C. et al. Activation of mammalian target of rapamycin in cytomegalic neurons of human cortical dysplasia. Ann. Neurol. 60, 420-429 (2006).

41. Miyata, H., Chiang, A. C. \& Vinters, H. V. Insulin signaling pathways in cortical dysplasia and TSC-tubers: tissue microarray analysis. Ann. Neurol. 56, 510-519 (2004).

42. Wong, M. Mammalian target of rapamycin (mTOR) activation in focal cortical dysplasia and related focal cortical malformations. Exp. Neurol. 244, 22-26 (2013).

43. Fisher, R. S. et al. Epileptic seizures and epilepsy: definitions proposed by the International League Against Epilepsy (ILAE) and the International Bureau for Epilepsy (IBE). Epilepsia 46, 470-472 (2005).

44. Nie, D. et al. Tsc2-Rheb signaling regulates EphA-mediated axon guidance. Nat. Neurosci. 13, 163-172 (2010).

45. Sato, A. et al. Rapamycin reverses impaired social interaction in mouse models of tuberous sclerosis complex. Nat. Commun. 3, 1292 (2012).

46. Vail, E. A., Rakowski, S. K., Numis, A. L. \& Thiele, E. A. Role of mutational analysis in diagnosis of tuberous sclerosis complex. Clin. Genet. 75, 282-285 (2009).
47. Chu-Shore, C. J., Major, P., Camposano, S., Muzykewicz, D. \& Thiele, E. A The natural history of epilepsy in tuberous sclerosis complex. Epilepsia 51, 1236-1241 (2010).

48. Beenhakker, M. P. \& Huguenard, J. R. Neurons that fire together also conspire together: is normal sleep circuitry hijacked to generate epilepsy? Neuron 62, 612-632 (2009).

49. Huguenard, J. R. \& McCormick, D. A. Thalamic synchrony and dynamic regulation of global forebrain oscillations. Trends Neurosci. 30, 350-356 (2007)

50. McCormick, D. A. \& Bal, T. Sleep and arousal: thalamocortical mechanisms. Annu. Rev. Neurosci. 20, 185-215 (1997).

51. Kandel, A. \& Buzsaki, G. Cellular-synaptic generation of sleep spindles, spike-and-wave discharges, and evoked thalamocortical responses in the neocortex of the rat. J. Neurosci. 17, 6783-6797 (1997).

52. Traub, R. D. et al. A possible role for gap junctions in generation of very fast EEG oscillations preceding the onset of, and perhaps initiating, seizures. Epilepsia 42, 153-170 (2001).

53. Lachaux, J. P. et al. The many faces of the gamma band response to complex visual stimuli. Neuroimage 25, 491-501 (2005).

54. Moddel, G. et al. The NMDA receptor NR2B subunit contributes to epileptogenesis in human cortical dysplasia. Brain. Res. 1046, 10-23 (2005)

55. Najm, I. M. et al. Epileptogenicity correlated with increased N-methyl-Daspartate receptor subunit NR2A/B in human focal cortical dysplasia. Epilepsia 41, 971-976 (2000).

56. Ghasemi, M. \& Schachter, S. C. The NMDA receptor complex as a therapeutic target in epilepsy: a review. Epilepsy Behav. 22, 617-640 (2011).

57. Liu, Y. et al. NMDA receptor subunits have differential roles in mediating excitotoxic neuronal death both in vitro and in vivo. J. Neurosci. 27, 2846-2857 (2007).

58. Krystal, J. H., Anand, A. \& Moghaddam, B. Effects of NMDA receptor antagonists: implications for the pathophysiology of schizophrenia. Arch. Gen. Psychiatry 59, 663-664 (2002).

59. Carlen, M. et al. A critical role for NMDA receptors in parvalbumin interneurons for gamma rhythm induction and behavior. Mol. Psychiatry 17, 537-548 (2012).

60. Cepeda, C., Andre, V. M., Vinters, H. V., Levine, M. S. \& Mathern, G. W. Are cytomegalic neurons and balloon cells generators of epileptic activity in pediatric cortical dysplasia? Epilepsia 46(Suppl 5): 82-88 (2005).

61. Aronica, E., Boer, K., Baybis, M., Yu, J. \& Crino, P. Co-expression of cyclin D1 and phosphorylated ribosomal S6 proteins in hemimegalencephaly. Acta Neuropathol. 114, 287-293 (2007).

62. Samadani, U., Judkins, A. R., Akpalu, A., Aronica, E. \& Crino, P. B. Differential cellular gene expression in ganglioglioma. Epilepsia 48, 646-653 (2007).

63. Wong, M. \& Crino, P. B. in mTOR and Epileptogenesis in Developmental Brain Malformations 4th edn (eds Noebels, J.L., Avoli, M., Rogawski, M.A., Olsen, R.W. \& Delgado-Escueta, A.V.) (National Center for Biotechnology Information, 2012).

64. Crino, P. B. Focal brain malformations: a spectrum of disorders along the mTOR cascade. Novartis Found. Symp. 288, 260-272 discussion 272-81 (2007).

65. Dayas, C. V., Smith, D. W. \& Dunkley, P. R. An emerging role for the Mammalian target of rapamycin in 'pathological' protein translation: relevance to cocaine addiction. Front. Pharmacol. 3, 13 (2012).

66. Han, J. M. \& Sahin, M. TSC1/TSC2 signaling in the CNS. FEBS Lett. 585, 973-980 (2011).

67. Landis, S. C. et al. A call for transparent reporting to optimize the predictive value of preclinical research. Nature 490, 187-191 (2012).

68. Clark, D. E. Rapid calculation of polar molecular surface area and its application to the prediction of transport phenomena. 2. Prediction of blood-brain barrier penetration. J. Pharm. Sci. 88, 815-821 (1999).

69. Cruciani, G., Pastor, M. \& Guba, W. VolSurf: a new tool for the pharmacokinetic optimization of lead compounds. Eur. J. Pharm. Sci. 11(Suppl 2): S29-S39 (2000).

70. Dedrick, R. L. \& Flessner, M. F. Pharmacokinetic problems in peritoneal drug administration: tissue penetration and surface exposure. J. Natl. Cancer. Inst. 89, 480-487 (1997).

\section{Acknowledgements}

This work was supported by funding from INSERM, Else Kröner-Fresenius Foundationproject number: 2010_A145 (A.J.B, N.B., C.C. and E.P.-P.); The French League Against Epilepsy (S.G.); The program of competitive growth of Kazan University and grant RFBR 13-04-01237a (M.Min.) and The French Association Sclérose Tubéreuse de Bourneville (N.B. and C.C.). We thank Thermalliance, Nerf and Dr Catherine Chiron for financial support (S.G. and N.L.) and Dr Diana Ferrari for critically reading the manuscript.

\section{Author contributions}

N.L. and T.T. performed electrophysiological experiments and data analysis in brain slices (patch-clamp); S.G., V.T., M.Min. and N.A.G. performed in vivo experiments and data analysis; V.T. performed extracellular recordings and data analysis in brain slices; 
A.B.-_Double-hit model' design and supervision; F.W., E.B. and S.G. performed electroporation and mutant mice handling; E.P.-P. and C.C. performed quantitative RT-PCR; M.Milh., D.S., C.B., G.D., S.G. and O.De. performed TSC patient's phenotyping analysis, surgery and clinical data collection; O.Du. and A.J.B. performed clinical and neuropathological analyses supervision; S.G., F.W. and V.T. performed mice genotyping; S.S. performed preliminary screen for drug efficacy; N.L., T.T., N.B. and S.G. performed experiments and data analysis in human samples; N.B. was the project leader; N.B., N.L., O.Du. and Y.B. performed experimental design; and N.B., N.L., O.Du., Y.B., A.R. and S.G. wrote the manuscript.

\section{Additional information}

Supplementary Information accompanies this paper at http://www.nature.com/ naturecommunications

Competing financial interests: On the 22 April 2014, Inserm Transfert filed a patent entitled 'Method and pharmaceutical composition for use in the treatment of epileptic seizures' (E.U. patent deposit number PCT/EP2014/058143); N.B., Y.B and N.L. are identified as inventors of this patent. All other authors declare no competing financial interests.

Reprints and permission information is available online at http://npg.nature.com/ reprintsandpermissions/

How to cite this article: Lozovaya, N. et al. Selective suppression of excessive GluN2C expression rescues early epilepsy in a tuberous sclerosis murine model. Nat. Commun. 5:4563 doi: 10.1038/ncomms5563 (2014).

(c) $(-)$ This work is licensed under a Creative Commons Attributioncc. ${ }_{\mathrm{BY}} \mathrm{NC}$ ND NonCommercial-NoDerivs 4.0 International License. The images or other third party material in this article are included in the article's Creative Commons license, unless indicated otherwise in the credit line; if the material is not included under the Creative Commons license, users will need to obtain permission from the license holder to reproduce the material. To view a copy of this license, visit http:// creativecommons.org/licenses/by-nc-nd/4.0/ 